UNIVERSIDADE DE SÃO PAULO
HOSPITAL DE REABILITAÇÃO DE ANOMALIAS CRANIOFACIAIS

\title{
AVALIAÇÃO DA MOBILIDADE VELAR EM INDIVÍDUOS COM INSUFICIÊNCIA VELOFARÍNGEA POR RINOMETRIA ACÚSTICA
}

BRUNA MARA ADORNO MARMONTEL ARAÚJO

Dissertação apresentada ao Hospital de Reabilitação de Anomalias Craniofaciais da Universidade de São Paulo para obtenção do título de MESTRE em Ciências da Reabilitação.

Área de concentração: Fissuras Orofaciais e Anomalias Relacionadas.

BAURU 



\author{
UNIVERSIDADE DE SÃO PAULO \\ HOSPITAL DE REABILITAÇÃO DE ANOMALIAS CRANIOFACIAIS
}

\title{
AVALIAÇÃO DA MOBILIDADE VELAR EM INDIVÍDUOS COM INSUFICIÊNCIA VELOFARÍNGEA POR RINOMETRIA ACÚSTICA
}

BRUNA MARA ADORNO MARMONTEL ARAÚJO

Orientadora: Prof ${ }^{\mathrm{a}}$. Dr ${ }^{\mathrm{a}}$. Inge Elly Kiemle Trindade

Dissertação apresentada ao Hospital de Reabilitação de Anomalias Craniofaciais da Universidade de São Paulo para obtenção do título de MESTRE em Ciências da Reabilitação.

Área de concentração: Fissuras Orofaciais e Anomalias Relacionadas.

BAURU 
AUTORIZO A REPRODUÇÃO E DIVULGAÇÃO DESTE TRABALHO, POR QUALQUER MEIO CONVENCIONAL OU ELETRÔNICO, PARA FINS DE ESTUDO E PESQUISA, DESDE QUE CITADA A FONTE.

Araújo, Bruna Mara Adorno Marmontel

A15a Avaliação da mobilidade velar em indivíduos com insuficiência velofaríngea por rinometria acústica / Bruna Mara Adorno Marmontel Araújo. Bauru, 2010. 102p.; il.; $30 \mathrm{~cm}$.

Dissertação (Mestrado - Área de Concentração: Fissuras Orofaciais e Anomalias relacionadas) - HRAC-USP

Orientador: Prof ${ }^{\mathrm{a}}$. Dra ${ }^{\mathrm{a}}$. Inge Elly Kiemle Trindade

1. Rinometria acústica. 2. Insuficiência velofaríngea. 3. Nasofaringe. 


\section{FOLHA DE APROVAÇÃO}

\section{Bruna Mara Adorno Marmontel Araújo}

Dissertação apresentada ao Hospital de Reabilitação de Anomalias Craniofaciais da Universidade de São Paulo para obtenção do titulo de Mestre em Ciências da Reabilitação.

Área de Concentração: Fissuras Orofaciais e Anomalias Relacionadas

Aprovado em:

Banca Examinadora

Prof. Dr.

Instituição

Assinatura

Prof. Dr.

Instituição Assinatura

Prof. Dr.

Instituição (Orientador):

Profa ${ }^{a}$. Dra . Inge Elly Kiemle Trindade

Presidente da Comissão de Pós-Graduação do HRAC-USP

Data do depósito da dissertação junto ao SPG: 



\section{Bruna Mara Adorno Marmontel Araújo}

25 de maio de 1981

Bauru-SP

$2000-2005$

2006-2006

$2007-2010$

2009 até a presente data
Nascimento

Graduação em Fonoaudiologia - Universidade do

Sagrado Coração (USC)

Fonoaudióloga da Prefeitura Municipal de

Paraipaba-CE

Curso de Pós-Graduação em Ciências da

Reabilitação, Área de Concentração: Fissuras

Orofaciais e Anomalias Relacionadas, Nível

Mestrado - Hospital de Reabilitação de Anomalias

Craniofaciais (HRAC) - USP

Fonoaudióloga do Laboratório de Fisiologia do Hospital de Reabilitação de Anomalias Craniofaciais (HRAC) - USP 



\section{DEDICATÓRIA}

Dedico este trabalho primeiramente a Deus, autor da minha vida, pois sem Ele, nada seria possível.

Aos dois grandes amores da minha vida:

Meu marido Bruno, por abrir mão dos seus sonhos para trilhar os meus sonhos, pelo amor incondicional, paciência e compreensão, fundamentais para a realização deste trabalho.

Minha querida filha Júlia, pela dádiva de ser sua mãe, por me tornar uma pessoa ainda mais feliz e realizada.

Dedico ainda, à minha mãe Cleide, pelo amor, carinho e dedicação com que me criou. Pelo apoio e compreensão nesta etapa da minha vida.

Ao querido Luiz, meu reconhecimento e gratidão pelo carinho de pai que sempre demonstrou.

À minha querida avó Zezé, que já não está entre nós, mas sempre será meu grande exemplo de vida. 

À minha orientadora,

\section{Profa. Dra. Inge Elly Kiemle Trindade}

Meu eterno agradecimento, pela confiança depositada em mim, mesmo sem me conhecer, por despertar em mim o interesse pela pesquisa, pelo cuidado $e$ competência na orientação deste trabalho. Minha profunda admiração e respeito pelo seu conhecimento, sabedoria e dedicação à ciência. 



\section{AGRADECIMENTOS}

Ao Hospital de Reabilitação de Anomalias Craniofaciais, da Universidade de São Paulo, na pessoa de seu Superintendente Prof. Dr. José Aberto de Souza Freitas.

À Comissão de Pós-Graduação do HRAC, na pessoa de sua presidente Prof ${ }^{a}$. Drª. Inge Elly Kiemle Trindade.

À Drạ. Renata Paciello Yamashita, pelo convívio diário, apoio e compreensão.

À Drà. Ana Claudia Martins Sampaio Teixeira, pelo convívio, carinho, amizade e contribuições fundamentais para realização deste trabalho.

À Drà. Ana Paula Fukushiro, pelo convívio diário e pela sua importante contribuição na Qualificação deste trabalho.

À amiga Cristiane Luz, pela profunda e sincera amizade que nasceu desde o primeiro dia em que nos conhecemos na sala de aula da Pós-Graduação do HRACUSP.

À amiga Marcelle Raia, pelo apoio e incentivo no início deste trabalho.

À Dra. Adriana Gomes, pela paciência e colaboração no início deste trabalho. 

Às amigas pós-graduandas Carolina Brustello, Daniela Barbosa, Daniela Modolo, Daniele Baraldi, Priscila Prado e Tatiane Romanini, pelas conversas e anseios compartilhados durante esses anos.

Às alunas da Prática Profissionalizante, Adriana e Gabriela, pelo apoio demonstrado na fase final deste trabalho.

À Secretaria do Programa de Pós-Graduação do HRAC-USP, nas pessoas de Andréia, Maria José (Zezé) e Rogério, pelo carinho, apoio e disponibilidade em todos os momentos necessários.

Ao Prof. Dr. José Roberto Pereira Lauris e Marcel Taga pela contribuição na análise estatística dos dados.

Aos pacientes e voluntários que participaram deste estudo.

A todos, que de alguma forma contribuíram para a realização deste trabalho. 



\section{RESUMO}

Araújo BMAM. Avaliação da mobilidade velar em indivíduos com insuficiência velofaríngea por rinometria acústica. [Dissertação]. Bauru: Hospital de Reabilitação de Anomalias Craniofaciais, Universidade de São Paulo; 2010.

Objetivo: Verificar se a rinometria acústica, usada de rotina para avaliar a patência nasal, é capaz de identificar a deficiência no movimento velar em indivíduos com diagnóstico clínico de função velofaríngea inadequada (FVI).

Modelo: Estudo clínico prospectivo

Local de Execução: Hospital de Referência Especializado

Participantes: Vinte indivíduos com fissura de palato reparada e FVI residual e 18 indivíduos-controle sem fissura de palato e função velofaríngea adequada (FVA), adultos, de ambos os sexos.

Variáveis analisadas: Curvas área-distância foram obtidas no repouso velar e na fala (fonema / $/ /$ ), utilizando um sistema Eccovision $A R$, sendo o volume determinado pela integração da área sob a curva em segmento correspondente à nasofaringe. $A$ mobilidade velar $(\Delta \mathrm{V})$ foi estimada pela diferença absoluta e relativa entre o volume nasofaríngeo no repouso velar $(V r)$ e na fala $(V k)$. A eficiência da técnica em discriminar FVI e FVA foi analisada pela curva ROC.

Resultados: Os valores médios ( $\pm D P$ ) de $V r$ e Vk obtidos foram: $23,2 \pm 3,6 \mathrm{~cm}^{3}$ e $15,9 \pm 3,8 \mathrm{~cm}^{3}$, no grupo FVA, e $22,7 \pm 7,9 \mathrm{~cm}^{3}$ e $20,7 \pm 7,4 \mathrm{~cm}^{3}$, no grupo $\mathrm{FVI}$, correspondendo a uma redução média de $7,3 \mathrm{~cm}^{3}(31 \%)$ no grupo FVA e a uma redução significativamente menor, de $2,0 \mathrm{~cm}^{3}(9 \%)$, no grupo $\mathrm{FVI}(p<0,05)$. Constatou-se que $70 \%$ dos pacientes do grupo $\mathrm{FVI}$ apresentaram $\Delta \mathrm{V}$ sugestivo de elevação velar prejudicada (inferior ao limiar de corte que maximizou, simultaneamente, a sensibilidade e a especificidade do teste), confirmando o diagnóstico clínico.

Conclusão: A rinometria acústica foi capaz de identificar, com bom poder discriminatório, o comprometimento da atividade velar que caracteriza a insuficiência velofaríngea.

Descritores: rinometria acústica, insuficiência velofaríngea, nasofaringe. 



\section{ABSTRACT}

Araújo BMAM. Assessment of velar mobility in individuals with velopharyngeal insufficiency by acoustic rhinometry. [Dissertation]. Bauru: Hospital de Reabilitação de Anomalias Craniofaciais, Universidade de São Paulo; 2010.

Objective: To determine whether acoustic rhinometry, routinely used for evaluation of nasal patency, is able to identify impairment of velar movement in individuals with clinical diagnosis of inadequate velopharyngeal function (IVF).

Design: Prospective clinical study.

Setting: Reference Craniofacial Hospital.

Participants: Twenty subjects with repaired cleft palate and residual IVF and 18 noncleft controls with adequate velopharyngeal function (AVF), adults, of both sexes.

Main Outcomes Measures: Area-distance curves were obtained during velar rest and speech (phoneme $/ \mathrm{k} /$ ), using an Eccovision $A R$ system, and volume was determined by integrating the area under the curve at a segment corresponding to nasopharynx. Velar mobility $(\Delta \mathrm{V})$ was estimated by the absolute and relative difference between nasopharyngeal volume at velar rest $(\mathrm{Vr})$ and speech $(\mathrm{Vk})$. The efficiency of the technique to discriminate IVF and AVF was assessed by a ROC curve.

Results: Mean $\mathrm{Vk}$ and $\mathrm{Vr}$ values $( \pm \mathrm{SD})$ obtained were: $23.2 \pm 3.6 \mathrm{~cm}^{3}$ and $15.9 \pm 3.8 \mathrm{~cm}^{3}$ (AVF group), and $22.7 \pm 7.9 \mathrm{~cm}^{3}$ and $20.7 \pm 7.4 \mathrm{~cm}^{3}$ (IVF group), corresponding to an average reduction of $7.3 \mathrm{~cm}^{3}(31 \%)$ for the AVF group and a significantly smaller reduction of $2.0 \mathrm{~cm}^{3}(9 \%)$ for the IVF group $(p<0.05)$. Seventy percent of the IVF patients showed a $\Delta \mathrm{V}$ suggesting impaired velar elevation (below the cutoff score that maximized both the sensitivity and specificity of the test), confirming clinical diagnosis.

Conclusion: Acoustic rhinometry was able to identify, with a good discriminatory power, the impairment of velar activity which characterizes velopharyngeal insufficiency.

Keywords: acoustic rhinometry, velopharyngeal insufficiency, nasopharynx. 



\section{LISTA DE FIGURAS}

Figura 1 - Rinômetro Acústico: (1) tubo do rinômetro com adaptador nasal (nosepiece); (2) tela do microcomputador com rinograma

Figura 2 - Rinômetro Acústico: representação esquemática da instrumentação usada para a medida das dimensões internas da cavidade nasal e da nasofaringe (adaptado de Roithmann e Cole 1995).

Figura 3 - Rinograma: área de secção tranversa nasal (eixo y) em função da distância relativamente à narina (eixo $\mathrm{x}$ ), correspondente a primeira deflexão. A segunda deflexão corresponde à área de secção transversa da válvula nasal, usada para a escolha da cavidade nasal de melhor permeabilidade (Gomes et al 2009).....

Figura 4 - $O$ traçado $(A)$ corresponde a dois rinogramas sobrepostos obtidos durante o repouso e a atividade velar (fala): a seta indica o ponto de divergência entre as curvas demonstrando elevação velar. Os traçados $(B)$ e $(C)$ correspondem aos dois rinogramas isoladamente, mostrando o segmento utilizado para a determinação do volume nasofaríngeo (Vk e Vr), calculado pela integração da área sob a curva.

Figura 5 - Comparação do volume nasofaríngeo observado nos indivíduos com função velofaríngea adequada (FVA) e inadequada (FVI). Em (A) são apresentados os volumes absolutos aferidos nos dois grupos, no repouso velar $(\mathrm{Vr})$ e na fala (produção do fonema $/ k /$ ) $(V k)$. Em (B) são comparados as diferenças absolutas entre $\mathrm{Vr}$ e $\mathrm{Vk}$ e em (C) as diferenças relativas entre $\mathrm{Vr}$ e Vk nos dois grupos. Os traços horizontais representam o valor mediano do volume nasofaríngeo (Me), o $1^{\circ}$ e o $3^{\circ}$ quartis (Q1 e Q3) e os valores máximo (max) e mínimo (min) observados desconsiderando os valores discrepantes (círculos) identificados conforme critério estabelecido por Tukey (1977).......................... 
Figura 6a - Valores absolutos do volume nasofaríngeo no repouso velar e na fala observados nos indivíduos com função velofaríngea adequada (FVA). O número mostrado acima e à direita do traçado corresponde a variação volumétrica observada $(\Delta \mathrm{VA}) \ldots \ldots$.

Figura 6b - Valores absolutos do volume nasofaríngeo no repouso velar e na fala observados nos indivíduos com função velofaríngea adequada (FVA). O número mostrado acima e à direita do traçado corresponde a variação volumétrica observada $(\triangle \mathrm{VA})$

Figura 6c - Valores absolutos do volume nasofaríngeo no repouso velar e na fala observados nos indivíduos com função velofaríngea adequada (FVA). O número mostrado acima e à direita do traçado corresponde a variação volumétrica observada $(\triangle \mathrm{VA})$

Figura 7a - Valores absolutos do volume nasofaríngeo no repouso velar e na fala observados nos indivíduos com função velofaríngea inadequada (FVI). Os indivíduos 1, 5 e 6 apresentaram resultado sugestivo de ausência de elevação velar. O número mostrado acima e à direita do traçado corresponde a variação volumétrica observada ( $\triangle \mathrm{VA})$

Figura 7b - Valores absolutos do volume nasofaríngeo no repouso velar e na fala observados nos indivíduos com função velofaríngea inadequada (FVI). Os indivíduos 8, 10 e 12 apresentaram resultado sugestivo de ausência de elevação velar. O número mostrado acima e à direita do traçado corresponde a variação volumétrica observada $(\triangle \mathrm{VA})$

Figura 7c - Valores absolutos do volume nasofaríngeo no repouso velar e na fala observados nos indivíduos com função velofaríngea inadequada $(\mathrm{FVI})$. Os indivíduos 13, 14, 15, 17 e 18 apresentaram resultado sugestivo de ausência de elevação velar. O número mostrado acima e à direita do traçado corresponde a variação volumétrica observada ( $\triangle \mathrm{VA})$ 
Figura 7d - Valores absolutos do volume nasofaríngeo no repouso velar e na fala observados nos indivíduos com função velofaríngea inadequada (FVI). Os indivíduos 19 e 20 apresentaram resultado sugestivo de ausência de elevação velar. O número mostrado acima e à direita do traçado corresponde a variação volumétrica observada $(\triangle \mathrm{VA})$

Figura 8 - Gráfico de dispersão ilustrando a mobilidade velar individual entre o grupo FVA e o grupo FVI. 



\section{LISTA DE QUADROS}

Quadro 1 - Vocábulos e sentenças utilizados na avaliação perceptiva da fala (Genaro et al 2004).

Quadro 2 - Classificação da função velofaríngea segundo escores de hipernasalidade, emissão de ar nasal e distúrbios articulatórios compensatórios (Trindade et al 2005). 



\section{LISTA DE TABELAS}

Tabela 1 - Volumes nasofaríngeos: valores médios \pm desvio padrão, valores mínimos e máximos, diferença absoluta $(\Delta \mathrm{VA})$ e relativa $(\Delta \mathrm{VR})$, observados no repouso velar e na fala, em indivíduos com a função velofaríngea adequada (FVA) e inadequada (FVI)

Tabela 2 - Volumes nasofaríngeos individuais: valores médios e diferença absoluta $(\triangle \mathrm{VA})$ e relativa $(\Delta \mathrm{VR})$, observados no repouso velar $e$ na a fala, em indivíduos com a função velofaríngea adequada (FVA)

Tabela 3 - Volumes nasofaríngeos individuais: valores médios e diferença absoluta $(\Delta \mathrm{VA})$ e relativa $(\Delta \mathrm{VR})$, observados no repouso velar $\mathrm{e}$ na fala, em indivíduos com a função velofaríngea inadequada (FVI).

Tabela 4 - Sensibilidade e especificidade da rinometria acústica como teste diagnóstico da disfunção velofaríngea para diferentes $\triangle \mathrm{VA}$ (diferença absoluta entre volume nasofaríngeo durante a produção do fonema $/ \mathrm{k} /$ e o repouso velar). O valor em negrito corresponde ao $\triangle \mathrm{VA}$ que maximizou a sensibilidade e a especificidade, simultaneamente, e, portanto, a ser considerado como valor de corte.

Tabela 5 - Sensibilidade e especificidade da rinometria acústica como teste diagnóstico da disfunção velofaríngea para diferentes valores de $\Delta \mathrm{VR}$ (diferença relativa entre volume nasofaríngeo durante a produção do fonema $/ \mathrm{k} /$ e o repouso velar). $\mathrm{O}$ valor em negrito corresponde ao $\Delta \mathrm{VR}$ que maximizou a sensibilidade e a especificidade, simultaneamente, e, portanto, a ser considerado como valor de corte 



\section{SUMÁRIO}

1 INTRODUÇÃO E REVISÃO DE LITERATURA ………………………......... 29

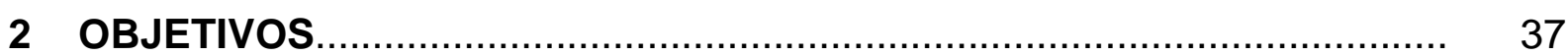

3 MATERIAL E MÉTODO ......................................................................... 41

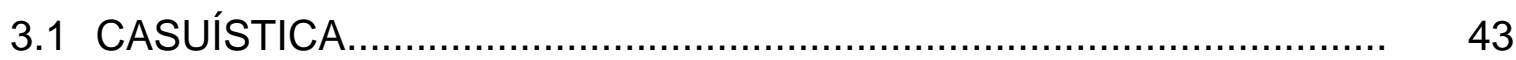

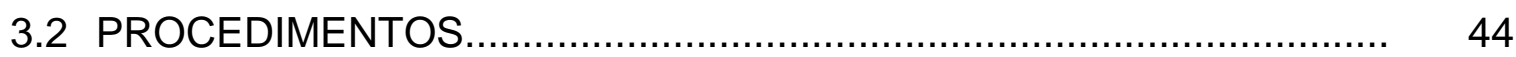

3.2.1 Avaliação perceptiva da função velofaríngea........................ 44

3.2.2 Avaliação da mobilidade velar por rinometria acústica......... 47

3.3 ANÁLISE ESTATÍSTICA........................................................... 54

4 RESULTADOS

4.1 ANÁLISE DOS VALORES MÉDIOS DO VOLUME NASOFARÍNGEO NO REPOUSO VELAR E NA FALA .................................................. 57

4.2 ANÁLISE DOS VALORES INDIVIDUAIS DO VOLUME NASOFARÍNGEO NO REPOUSO VELAR E NA FALA........................ 60

4.3 ANÁLISE DA SENSIBILIDADE E ESPECIFICIDADE DA RINOMETRIA ACÚSTICA ........................................................... 71

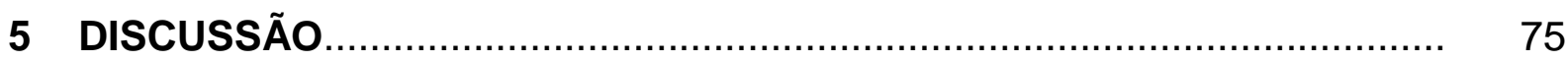

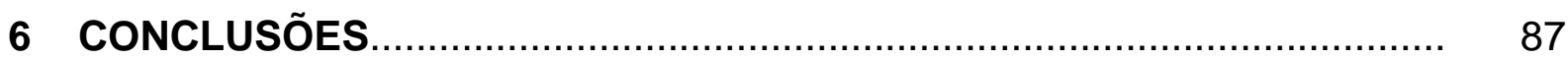

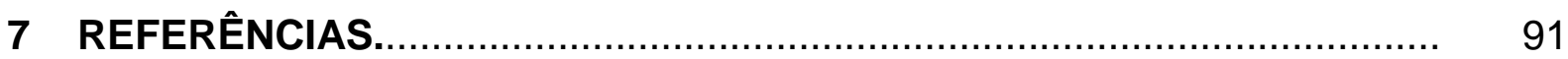

ANEXOS 



\section{INTRODUÇÃO E REVISÃO DE LITERATURA}





\section{INTRODUÇÃO E REVISÃO DE LITERATURA}

As fissuras palatinas são malformações congênitas que se estabelecem na vida intra-uterina durante o primeiro trimestre da gestação (Silva Filho e Freitas 2007). Podem comprometer estruturas importantes para produção de uma fala normal, como aquelas envolvidas no mecanismo velofaríngeo, responsável pela separação das cavidades oral e nasal durante a fala e em atividades como a respiração, a deglutição e o sopro (Kummer 2001).

O mecanismo velofaríngeo compreende o palato mole, as paredes laterais e a parede posterior da faringe, estruturas que delimitam um orifício que permanece aberto durante a produção de sons nasais, assim como na respiração nasal, e que se fecha durante a produção dos sons orais. Controla, dessa forma, o direcionamento do fluxo aéreo para cavidade nasal ou oral, levando a uma ressonância equilibrada durante a fala (Trindade et al 2005). O principal músculo responsável pela atividade velofaríngea é o levantador do véu palatino, que se origina na porção petrosa do osso temporal. Na respiração de repouso, o véu do palato mole mantém-se relaxado e na produção de sons orais se eleva posterior e superiormente em direção à parede posterior da faringe, sendo que a porção medial do véu do palato é a que usualmente mais se eleva, enquanto que o contato com a parede posterior da faringe é tipicamente realizado pelo terceiro quadrante. Já, na presença de uma fissura no palato, dois aspectos anatômicos caracterizam o mecanismo velofaríngeo, que podem levar ao que chamamos de falha no fechamento velofaríngeo: falta de aponeurose palatina na região anterior e músculos longitudinalmente inseridos na borda posterior do palato duro, inclusive o levantador do véu palatino (Moon e Kuehn 2004). 
A falha no fechamento velofaríngeo é designada genericamente como disfunção velofaríngea (DVF). O termo incompetência velofaríngea é usado especificamente quando a falha é de origem funcional e o termo insuficiência velofaríngea (IVF), foco de atenção do presente estudo é utilizado quando não ocorre o fechamento por fatores estruturais, ou seja, como decorrência de alterações anatômicas nas estruturas da velofaringe (Trost-Cardamone 1989).

A principal causa da IVF é, portanto, a própria fissura palatina. No entanto, essa condição pode persistir mesmo após a correção cirúrgica primária do palato (Fukushiro 2007), como resultado de um palato curto, de fibrose cicatricial ou de músculos posicionados incorretamente durante a cirurgia primária (Carvalho 2006, Rocha 2007). A IVF pode também resultar de palato curto e faringomegalia congênitos ou manifestar-se após adenoidectomia e/ou amigdalectomia, ressecções tumorais, uvuloplastia (Kummer 2001, Rocha 2007).

Quando as estruturas do mecanismo velofaríngeo falham em realizar o fechamento entre as duas cavidades durante a produção dos sons orais, parte da corrente aérea sonorizada é desviada para cavidade nasal, comprometendo a produção da fala de diferentes formas (Genaro et al 2007). Os sintomas mais comuns são a hipernasalidade, a emissão de ar nasal, a fraca pressão intra-oral, os movimentos faciais associados e os distúrbios articulatórios compensatórios (Golding-Kushner 1995, Kummer 2001, Peterson-Falzone et al 2001, TrostCardamonne 2004, Genaro et al 2007, Trindade et al 2007a).

O julgamento perceptivo da fala é o principal método de avaliação da IVF. No entanto, ainda que seja possível minimizar sua subjetividade, é concenso que o julgamento perceptivo está sujeito a variações e erros, mesmo entre ouvintes treinados, pois pode ser influenciado por fatores como padrão articulatório, 
tonalidade e intensidade da voz, contexto fonético, experiência prévia e expectativas do julgador (Couniham e Cullinan 1970, Dalston 2004, Trindade et al 2007a). Assim, para confirmar as impressões clínicas resultantes da avaliação perceptiva da fala, diferentes métodos instrumentais de diagnóstico vêm sendo utilizados para identificar a IVF e também para acompanhar os resultados das intervenções terapêuticas. A American Cleft Palate Association publicou um documento na década de 80 (Dalston et al 1988), recomendando que a avaliação de resultados cirúrgicos em indivíduos com fissura deve fundamentar-se em, pelo menos, um dos seguintes métodos instrumentais: nasofaringoscopia, videofluoroscopia, nasometria e rinomanometria, esta última também conhecida como técnica fluxo-pressão. A nasofaringoscopia e a videofluoroscopia são considerados métodos diretos de avaliação da função velofaríngea, uma vez que fornecem informações sobre a anatomia funcional da região velofaríngea. Já a nasometria e a rinomanometria são considerados métodos indiretos, pois aferem, respectivamente, os resultados acústicos e aerodinâmicos da atividade velofaríngea, ou seja, aferem o estado funcional do mecanismo velofaríngeo, fornecendo dados quantitativos que contribuem para a comparação dos resultados terapêuticos (Trindade et al 2007a).

Outro método instrumental proposto na literatura para avaliar a atividade velofaríngea é a rinometria acústica (Dalston 1992 e Seaver et al 1995). Descrita por Sondhi e Gopinath em 1971, a técnica foi inicialmente utilizada para a avaliação das dimensões internas da traquéia e árvore brônquica de cães por Jackson et al (1977). Atualmente, é utilizada, de rotina, para determinar áreas seccionais e volumes da cavidade nasal e, assim, caracterizar a geometria nasal (Hilberg e Pedersen 2000).

A técnica consiste na análise dos sons refletidos pela cavidade nasal em resposta a uma onda sonora incidente emitida por uma fonte sonora posicionada no 
interior de um tubo, que é encostado em uma das narinas (Hilberg e Pedersen 2000, Hilberg 2002). Os sinais de pressão sonora são captados por um microfone, também posicionados no interior do tubo, e analisados por um software que permite a determinação das áreas seccionais nasais, visualizadas em um gráfico áreadistância - o chamado "rinograma". Os volumes de diferentes segmentos da cavidade nasal e da nasofaringe podem também ser medidos a partir da integração da curva área-distância. Em suma, a rinometria acústica realiza medições consecutivas de toda a cavidade nasal, desde as narinas até a nasofaringe, permitindo a localização exata das constrições que determinam a resistência nasal (Hilberg et al 1989, Roithmann e Cole 1995, Bertier e Trindade 2007).

A rinometria acústica é, hoje, amplamente reconhecida como técnica de avaliação da permeabilidade nasal. Valores de referência de áreas seccionais e volumes, assim como valores anormais relacionados à obstrução das vias aéreas nasais de diferentes etiologias foram relatados por vários autores, validando o seu uso como método de avaliação das dimensões nasais (Gomes 2004, Prado 2009). Valores normativos também foram definidos no Laboratório de Fisiologia do HRAC/USP, para segmentos específicos da cavidade nasal (Trindade et al 2007b, Gomes et al 2008), bem como se estudou os efeitos da obstrução nasal sobre aquelas variáveis (Prado 2009, Trindade et al 2009).

O fato da rinometria acústica também permitir a mensuração das dimensões da nasofaringe fez com que Dalston (1992), em revisão publicada sobre as aplicações da técnica, propusesse seu uso para monitorar mudanças do volume nasofaríngeo durante a fala. A seguir, Seaver et al (1995) demonstraram que a rinometria acústica foi capaz de detectar mudanças na posição do véu do palato durante a fala em dois pacientes analisados. Comprovaram isto, superpondo os 
rinogramas obtidos no repouso velar e na produção contínua do fonema /f/, ao observarem o distanciamento das duas curvas, traduzindo a redução do volume nasofaríngeo decorrente da elevação velar.

É preciso ressaltar, contudo, que a medida da nasofaringe pela técnica rinométrica tem sido questionada pela interferência de fatores como: reflexão acústica para cavidade nasal contralateral ou seios paranasais, que pode superestimar as medidas (Kunkel e Hochban 1994a,b, Kunkel et al 1998a, Terheyden et al 2000, Cakmak et al 2005); presença de obstruções significativas situadas anteriormente à nasofaringe, que subestimaria as medidas (Terheyden et al 2000, Cakmak et al 2005), movimentos palatinos voluntários ou involuntários e esforço respiratório (Hilberg et al 1993, Fisher et al 1995, Clement e Gordts 2005, Prado 2009). Assim sendo, Kunkel et al (1998a,b), propuseram a análise da diferença entre o volume nasofaríngeo no repouso e o volume nasofaríngeo durante a fala, ou seja, $\Delta \mathrm{V}$, como indicador da mobilidade velar, o que por princípio, anula eventuais interferências causadas por esses fatores.

Kunkel et al (1998b), apresentaram evidências experimentais de que a rinometria acústica é, de fato, capaz de identificar variações volumétricas na nasofaringe. Primeiramente, utilizaram um balão inflável ligado a um catéter, que era inserido na cavidade nasal de voluntários normais. As medidas rinométricas eram, então, realizadas na cavidade nasal contralateral antes e após a insuflação do balão. Comparando a redução do volume nasofaríngeo causada pelo balão, medida pela rinometria acústica, e o volume de água usado para insuflar o balão, os autores observaram excelente coeficiente de correlação entre as variáveis $(0,93)$. A partir dos dados obtidos, concluíram, uma vez mais, que a técnica se mostrava adequada 
para avaliar, quantitativamente, as mudanças que ocorrem no volume da nasofaringe durante a fala.

Seguindo esse raciocínio, examinaram, no mesmo trabalho, indivíduos com fissura de palato supostamente reparada cirurgicamente, comparando-os com indivíduos sem fissura de palato, sendo que parte deles apresentava retalho faríngeo. Para estimar a mobilidade velar, o volume nasofaríngeo foi determinado durante o repouso, após alguns ciclos de respiração profunda, e durante o fechamento velofaríngeo, na produção sustentada do fonema /k/. Verificaram que a mobilidade velar máxima observada no grupo de pacientes com fissura se mostrou significantemente menor que a observada no grupo controle. Esses dados foram apresentados para uma amostra ligeiramente diversa em outra publicação do grupo (Kunkel et al 1998a).

Em estudo mais recente, conduzido no Laboratório de Fisiologia do HRAC/USP, Gomes (2004) demonstrou em indivíduos sem fissura, que o volume nasofaríngeo sofre redução de cerca de $20 \%$ como resultado da elevação velar na produção contínua da fricativa /f/, quando comparado ao do repouso.

Os poucos estudos publicados na área, vão de encontro, ao sugerido por Dalston (1992), de que a rinometria acústica é um método capaz de avaliar a mobilidade velar em pacientes com IVF. Porém, conforme salientado por Seaver et al (1995), outros estudos devem ainda ser realizados, para validar esse importante instrumento na prática clínica, especialmente porque se trata de um exame objetivo, não invasivo e que não causa desconforto ao paciente. Se comprovado que a rinometria acústica realmente é capaz de identificar a deficiência do movimento velar, esta técnica se tornará uma importante ferramenta não só no diagnóstico da DVF, como também no acompanhamento da terapia fonoaudiológica. 
2 OBJETIVOS 



\section{OBJETIVOS}

O presente estudo teve como objetivo principal determinar se a rinometria acústica é capaz de identificar a deficiência no movimento velar, em indivíduos com fissura de palato reparada, e diagnóstico de função velofaríngea inadequada, comparativamente a indivíduos sem fissura de palato e função velofaríngea adequada, segundo julgamento perceptivo.

Os objetivos específicos do estudo foram:

1. Determinar o volume nasofaríngeo no repouso velar e durante a fala;

2. Analisar a mobilidade velar, pelo cálculo da variação volumétrica da nasofaringe na fala;

3. Comparar o movimento velar de indivíduos com e sem IVF;

4. Analisar a sensibilidade e a especificidade da técnica na avaliação da função velofaríngea. 

3 MATERIAL E MÉTODO 



\section{MATERIAL E MÉTODO}

\subsection{CASUÍSTICA}

Foram submetidos à avaliação perceptiva da função velofaríngea, 31 pacientes com fissura de palato operada, associada ou não à fissura de lábio, não sindrômicos, em atendimento regular no Laboratório de Fisiologia do Hospital de Reabilitação de Anomalias Craniofaciais (HRAC-USP). Desse total, 20 pacientes com idade entre 17 e 35 anos (média de 24 anos), sendo 13 do sexo feminino e 7 do sexo masculino, foram classificados como tendo função velofaríngea inadequada (FVI), e, portanto, considerados aptos a compor o grupo experimental. No estudo, não foram incluídos pacientes submetidos à cirurgia de retalho faríngeo.

Para fins de controle, foram avaliados 18 voluntários, com idade entre 20 e 35 anos (média de 26 anos), sendo 12 do sexo feminino e 6 do sexo masculino, sem fissura de lábio ou palato e com função velofaríngea adequada (FVA). Foram excluídos do estudo, indivíduos com incapacidade física e/ou mental para realização dos exames, fístulas residuais extensas de palato e obstrução nasal no momento do exame, decorrente de processos respiratórios alérgicos, sendo agudos ou crônicos.

O estudo recebeu aprovação do Comitê de Ética em Pesquisa do HRAC/USP, conforme ofício n² 248/2007 (Anexo 1), sendo que os exames foram realizados após assinatura do termo de consentimento livre e esclarecido (Anexo 2). 


\subsection{PROCEDIMENTOS}

\subsubsection{Avaliação perceptiva da função velofaríngea}

A avaliação perceptiva foi realizada pela pesquisadora principal sob supervisão de fonoaudióloga experiente, conforme descrito por Genaro et al (2004). Foram avaliadas amostras de fala durante a conversa espontânea e repetição de sentenças e vocábulos foneticamente balanceados, mostrados no quadro 1, com a finalidade de identificar alterações relacionadas a IVF: hipernasalidade, emissão de ar nasal e distúrbios articulatórios compensatórios. A classificação da função velofaríngea foi feita utilizando o protocolo estruturado proposto por Trindade et al (2005), mostrado no quadro 2, baseado na atribuição de escores. A hipernasalidade foi classificada em uma escala de 6 pontos, na qual 1=ausente, $2=$ leve, $3=$ leve para moderada, 4=moderada, 5=moderada para grave e 6=grave. Foram considerados clinicamente significativos os escores iguais ou superiores a dois. A emissão de ar nasal, detectada no teste do espelho de Glatzel, colocado sob as narinas do paciente, foi classificada em escala de 6 pontos, na qual $1=$ ausente, $2=l e v e, 3=l e v e$ para moderada, 4=moderada, $5=$ moderada para grave e $6=$ grave. Foram considerados clinicamente significativos escores iguais ou superiores a três. As articulações compensatórias foram classificadas em uma escala de 2 pontos, na qual 1=ausentes e 2=presentes. Dependendo da combinação de escores observados para hipernasalidade, emissão nasal e articulações compensatórias, a função velofaríngea foi, então, classificada numa escala de três pontos, onde 1=adequada, 2=marginal e 3=inadequada. Os pacientes diagnosticados com função velofaríngea inadequada foram considerados aptos a participarem do estudo. 
Quadro 1 - Vocábulos e sentenças utilizados na avaliação perceptiva da fala (Genaro et al 2004).

\author{
Fonemas \\ /p/ - papai, pipa, apito \\ $/ \mathrm{t} / \mathrm{-}$ tatu, teto, ateu \\ $/ \mathrm{k} /$ - caqui, casa, aqui \\ /b/ - bebê, bola, abóbora \\ /d/ - dado, dedo, adoro \\ $/ \mathrm{g} /$ - garrafa, gole, agora \\ /m/ - mamãe, menino, amanhã \\ /n/ - nenê, nada, nunca \\ /y/ - ninho, passarinho, minhoca \\ /f/ - fita, Fábio, afiado \\ /s/ - saci, sítio, assado \\ I/ - Chico, chave, achado \\ /v/ - vovó, velho, uva \\ /z/ - zebra, zíper, azul \\ /J/ - jeito, jipe, caju \\ /// - lata, bala, alado \\ $/ N /$ - palha, palhaço, calha \\ $/ \mathrm{r} / \mathrm{\text {-barata}}$, amarelo, arara \\ /R/ - rato, rua, carroça
}

\section{Arquifonemas}

$\{R\}$ - carne, porta

$\{S\}$ - pasta, peste

Grupos consonantais:

planta, blusa

braço, prato

\section{Frases}

Papai pediu pipoca

A toca é do tatu

Cacá cortou o cabelo

A babá beijou o bebê

$O$ dedo do Dito doeu

Gugu gosta do gato

Mamãe comeu mamão

O nenê nada na piscina

O passarinho está no ninho

A fita da fada é de filó.

O saci sabe assobiar

Chico chupa chupeta.

Vovó viu o vestido

A casa da Zezé é azul

O jipe é do Juca

Lia lambeu o limão.

O palhaço olhou a ilha

A arara é amarela.

O rato roeu a roupa
Fonemas africados /1/ -

$/ \mathrm{t} \mathrm{J} /$ - titia, time, Tiago $/ \mathrm{r} /$ -

/d]/ - dia, Adilson, dinheiro 
Quadro 2 - Classificação da função velofaríngea segundo escores de hipernasalidade, emissão de ar nasal e distúrbios articulatórios compensatórios (Trindade et al 2005).

\begin{tabular}{|c|c|c|c|}
\hline \multicolumn{3}{|c|}{ Características da fala } & \multirow[b]{2}{*}{$\begin{array}{c}\text { Função } \\
\text { velofaríngea }\end{array}$} \\
\hline Hipernasalidade * & $\begin{array}{c}\text { Emissão de } \\
\text { ar nasal * }\end{array}$ & $\begin{array}{c}\text { Articulações } \\
\text { compensatórias \# }\end{array}$ & \\
\hline 1 & 1 & 1 & 1 = adequada \\
\hline 1 & 2 & 1 & $1=$ adequada \\
\hline 1 & 3 & $1-2$ & $2=$ marginal \\
\hline 2 & $1-3$ & $1-2$ & $2=$ marginal \\
\hline 3 & $1-3$ & $1-2$ & $2=$ marginal \\
\hline 2 & $4-6$ & $1-2$ & $3=$ inadequada \\
\hline 3 & $4-6$ & $1-2$ & $3=$ inadequada \\
\hline $4-6$ & $2-6$ & $1-2$ & $3=$ inadequada \\
\hline
\end{tabular}

* $1=$ ausente, $2=$ leve, $3=$ leve para moderada, $4=$ moderada, $5=$ moderada para grave, $6=$ grave. \# 1=ausente, $2=$ presente. 


\subsubsection{Avaliação da mobilidade velar por rinometria acústica}

\section{Equipamento e princípio da técnica}

A avaliação rinométrica foi realizada utilizando um sistema Eccovision Acoustic Rhinometer (HOOD Laboratories), mostrado na Figura 1. A técnica baseiase na análise de ondas sonoras refletidas (ecos) que emergem da cavidade nasal em resposta a uma onda sonora incidente. O sistema é composto por um tubo de aproximadamente $24 \mathrm{~cm}$, que tem em sua porção distal (relativamente ao indivíduo examinado), uma fonte sonora (alto-falante), e, na sua porção proximal, um microfone de registro. Durante o exame, a ponta do tubo, protegida por uma peça de silicone (nosepiece), é encostada em uma das narinas. A onda sonora gerada pelo alto-falante se propaga pelo tubo, passa pelo microfone, sem sensibilizá-lo, e entra na cavidade nasal. Variações da área de secção transversa, ou seja, constrições encontradas ao longo da cavidade nasal e nasofaríngea causam reflexões da onda sonora de volta ao tubo do rinômetro. Essas ondas sonoras refletidas sensibilizam o microfone, sendo então, amplificadas e digitalizadas. Um microcomputador com software específico é utilizado para a análise dos dados. A configuração do sistema é mostrada, esquematicamente, na Figura 2.

A área de secção transversa dos diferentes segmentos nasais desde a narina até a nasofaringe é calculadas pelo software a partir da intensidade do eco. A distância dos segmentos relativamente às narinas é calculada com base na velocidade da onda e o tempo de chegada do eco. Os dados são convertidos em função área-distância e representados na tela do computador na forma de um gráfico, o rinograma, no qual a área $\left(\mathrm{em} \mathrm{cm}^{2}\right)$ é apresentada em escala semilogarítmica no eixo y e a distância (em $\mathrm{cm}$ ) no eixo $x$ (Figura 3). A partir daí, os volumes podem ser calculados pela integração da área sob a curva. 


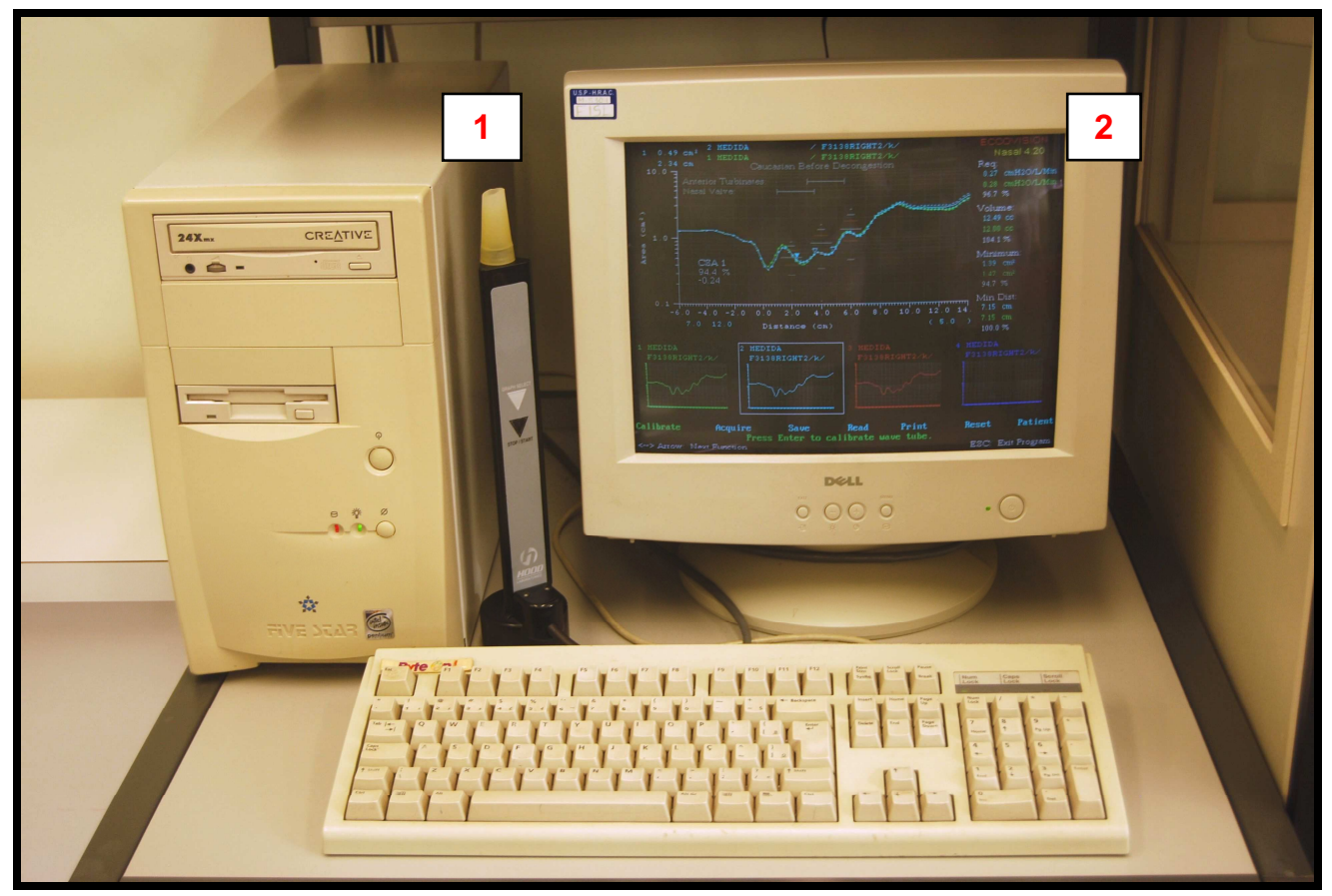

Figura 1 - Rinômetro Acústico: (1) tubo do rinômetro com adaptador nasal (nosepiece); (2) tela do microcomputador com rinograma. 


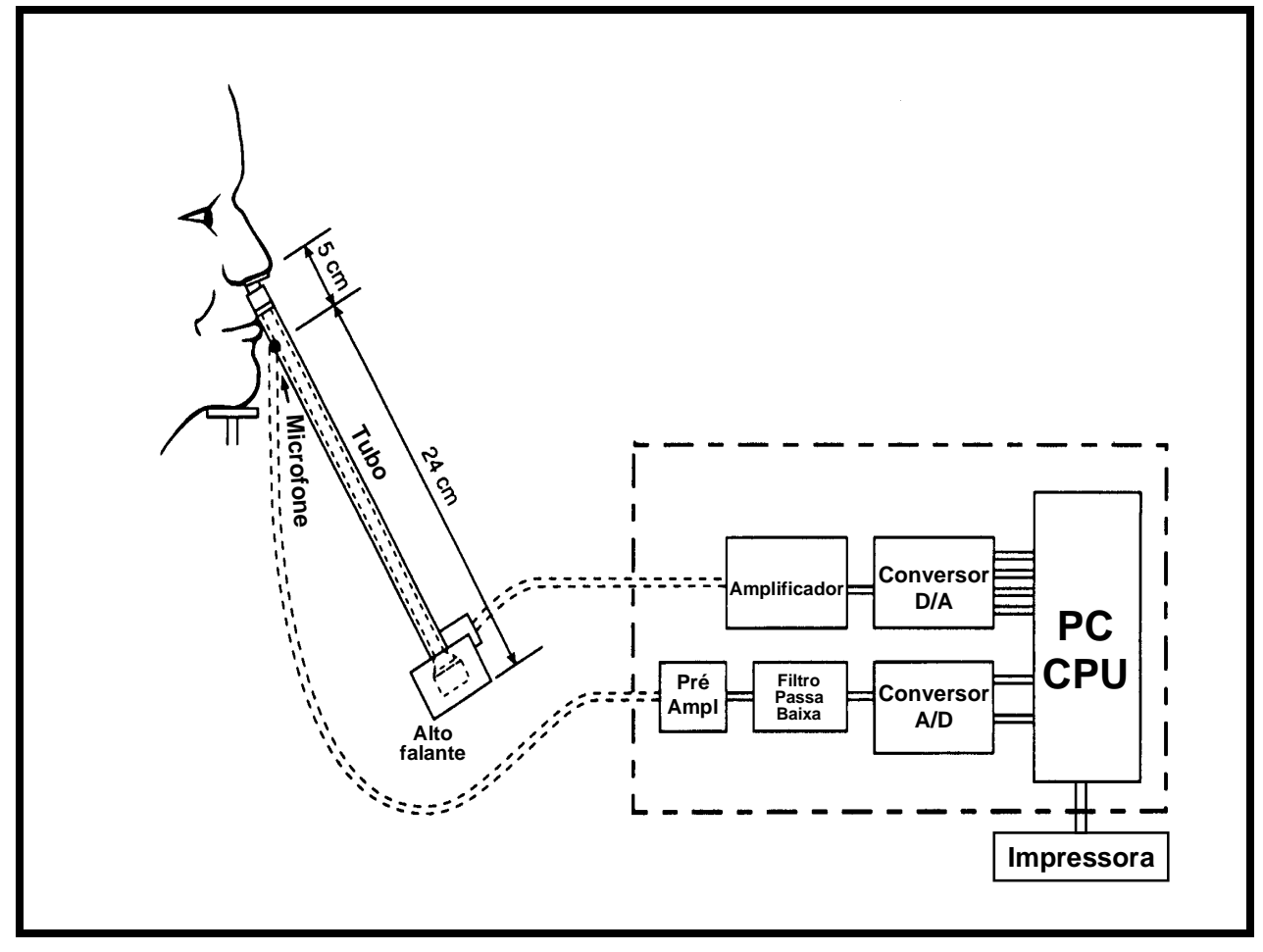

Figura 2 - Rinômetro Acústico: representação esquemática da instrumentação usada para a medida das dimensões internas da cavidade nasal e da nasofaringe (adaptado de Roithmann e Cole 1995). 


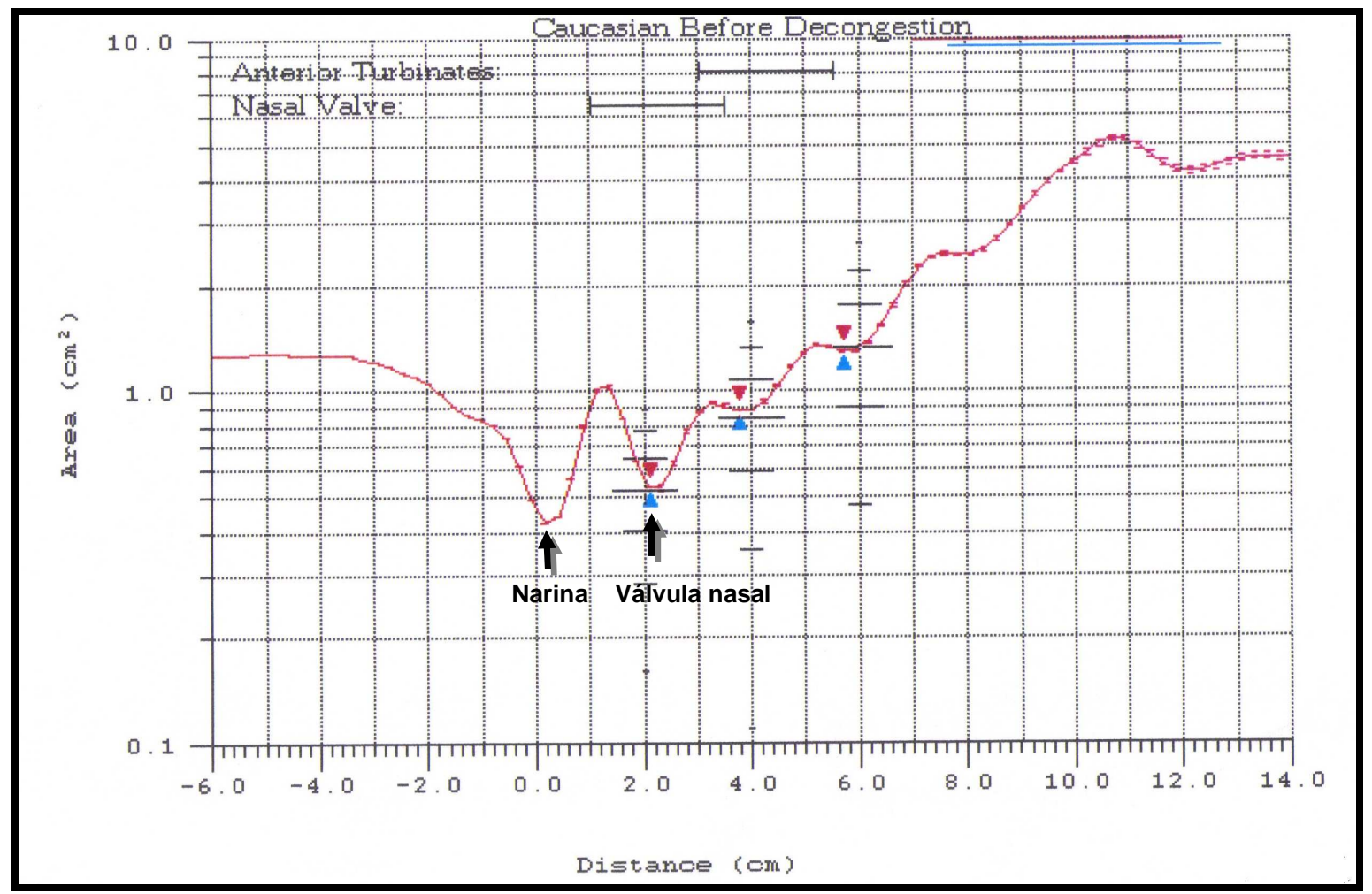

Figura 3 - Rinograma: área de secção tranversa nasal (eixo y) em função da distância relativamente à narina (eixo $\mathrm{x}$ ), correspondente a primeira deflexão. A segunda deflexão corresponde à área de secção transversa da válvula nasal, usada para a escolha da cavidade nasal de melhor permeabilidade (Gomes et al 2008). 
O sistema faz, a cada teste, medições em rápida sucessão (10 pulsos sonoros, são gerados aproximadamente a cada 0,5 segundos) e permite medidas de toda a cavidade nasal, dos lados direito e esquerdo independentemente, sendo que o software calcula a média das áreas seccionais e volumes das dez repetições.

\section{Procedimento}

O exame foi realizado em duas etapas, a primeira reproduzindo uma situação de relaxamento do véu do palato (repouso) e a segunda, a atividade velar máxima (fala):

- Etapa a (repouso): Uma vez posicionado o tubo do rinômetro, e após alguns ciclos respiratórios de repouso, o paciente era solicitado a suspender voluntariamente a respiração ao final de uma expiração e o sistema era, então, acionado para a aquisição dos dados. Três repetições eram feitas em cada cavidade nasal, sendo escolhido, para a análise do volume nasofaríngeo, o lado de melhor permeabilidade, ou seja, aquele com maior média da área seccional da válvula nasal (segunda deflexão do rinograma, conforme detalhado em Gomes et al 2008 (Figura 3).

- Etapa b (atividade): A seguir, o paciente era solicitado a produzir a palavra/kasa/, prolongando a fase de imposição da pressão oral do fonema $/ \mathrm{k} /$ por cerca de 5 segundos, e portanto, mantendo a tensão muscular até o término da aquisição dos dados.

Como mostra a Figura 4, no grupo FVA (controle), o volume nasofaríngeo foi determinado pela integração da curva área-distância, desde o ponto de divergência entre as curvas do repouso velar e da fala, correspondente à região das coanas (ou borda posterior do palato duro), até $5 \mathrm{~cm}$ a partir desse ponto, conforme realizado por Kunkel al (1998a). No grupo FVI (experimental), não tendo sido observado com nitidez o ponto de divergência entre as curvas em 8 casos, em função da falta de mobilidade velar, adotou-se como ponto inicial a distância média observada no 
(A)

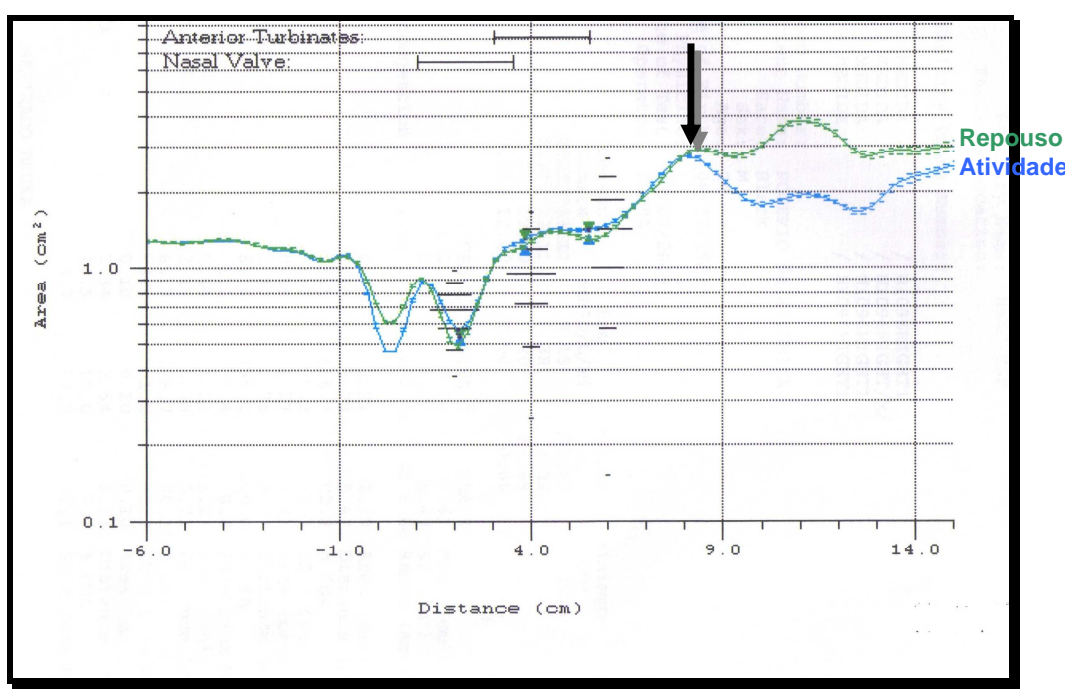

(B)

(C)
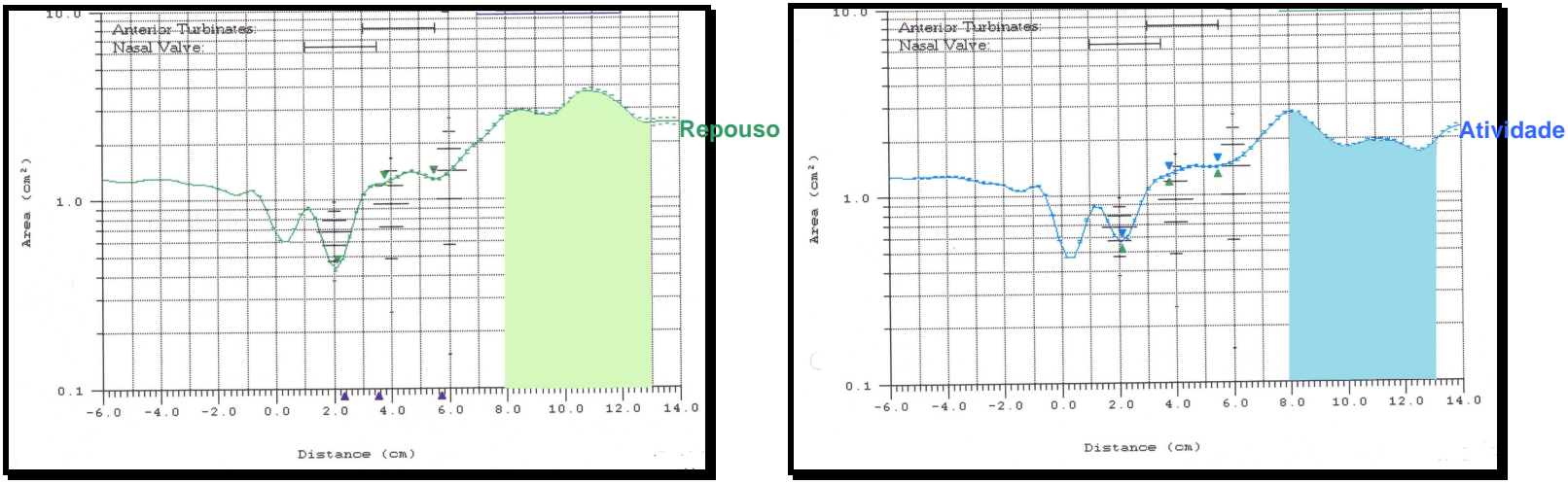

Figura 4 - $\mathrm{O}$ traçado $(\mathrm{A})$ corresponde a dois rinogramas sobrepostos obtidos durante 0 repouso e a atividade velar (fala): a seta indica o ponto de divergência entre as curvas demonstrando elevação velar. Os traçados $(B)$ e $(C)$ correspondem aos dois rinogramas isoladamente, mostrando o segmento utilizado para a determinação do volume nasofaríngeo (Vk e Vr), calculado pela integração da área sob a curva. 
grupo controle, que correspondeu a $7,7 \mathrm{~cm}$ a partir das narinas. Assim sendo, neste último grupo, o volume nasofaríngeo foi calculado para o intervalo entre 7,7 até $12,7 \mathrm{~cm}$ em relação às narinas. Portanto, em ambos os grupos, analisou-se um segmento de $5 \mathrm{~cm}$ a partir das coanas, representando a nasofaringe.

A mobilidade velar $(\Delta \mathrm{V})$ foi determinada calculando-se a diferença entre 0 volume nasofaríngeo no repouso velar $(\mathrm{Vr})$ e o volume nasofaríngeo durante a produção do fonema /k/ (Vk), ou seja, $\mathrm{Vk}$ - Vr, que corresponde à diferença absoluta $(\Delta V A)$, e, a razão entre $\mathrm{Vk}-\mathrm{Vr} / \mathrm{Vr}$, que corresponde à diferença relativa $(\Delta \mathrm{VR})$.

A calibração do equipamento foi feita a cada período do dia e com o intuito de minimizar possíveis erros nas medidas, os exames foram realizados sempre na mesma sala, em ambiente com temperatura relativamente estável e nível de ruído não superior a $60 \mathrm{db}$, após um período de 30 minutos de adaptação do paciente às condições ambientais. O tubo do rinômetro foi sempre posicionado em paralelo ao dorso do nariz e o vedamento entre o adaptador nasal (nosepiece) e a cavidade nasal foi assegurado pelo uso do gel neutro para eletrocardiograma a fim de evitar a perda sonora. Além disso, o paciente realizava o exame na posição sentada, com o mento e a testa apoiados em um suporte especialmente projetado para este fim, montado em uma haste fixada à cadeira. Quando os exames eram realizados durante a suspensão da respiração, os pacientes eram orientados a permanecer com a boca fechada, sem deglutir ou movimentar a língua no momento da aquisição dos dados, para evitar que a respiração e a deglutição interferissem nas medidas e na qualidade dos rinogramas. Tomou-se também o cuidado de não deformar a narina, e, portanto, a válvula nasal, durante o procedimento (Gomes 2004, Gomes 2007, Trindade et al 2007b, Gomes et al 2008, Trindade et al 2010). No grupo experimental, fístulas residuais de palato foram vedadas com retalho de hóstia, quando necessário. 


\subsection{ANÁLISE ESTATÍSTICA}

Considerando que a variável volume segue distribuição normal, com apenas discreto desvio à esquerda (Corey et al 1998), os resultados da rinometria acústica são expressos como média \pm desvio padrão.

Para analisar a significância das diferenças entre os volumes nasofaríngeos observados nas duas condições analisadas - produção do fonema $/ \mathrm{k} /$ e repouso velar - e nos dois grupos - função velofaríngea adequada e inadequada - utilizou-se a análise de variância a dois critérios modelo misto (Verbeke e Molenberghs 1997). As comparações post hoc foram feitas pelo teste de Tukey. Para a comparação do $\Delta \mathrm{V}$ entre os dois grupos foi utilizado o teste " $\mathrm{t}$ " de Student. Em todos os testes foi adotado o nível de significância de 5\% $(p<0,05)$.

Para o cálculo da sensibilidade e especificidade da técnica como teste diagnóstico, utilizou-se a análise da curva ROC (Receiver Operating Characteristic), para diferentes valores de $\Delta \mathrm{V}$ (diferença absoluta e relativa). Os valores máximos de sensibilidade e especificidade, obtidos simultaneamente, foram considerados como representativos da eficiência da rinometria acústica em identificar, respectivamente, a deficiência e a adequação da mobilidade velar (Vig et al 1991, Park et al 2004). 


\section{RESULTADOS}





\section{$4 \quad$ RESULTADOS}

\subsection{ANÁLISE DOS VALORES MÉDIOS DO VOLUME NASOFARÍNGEO NO REPOUSO VELAR E NA FALA}

Os valores médios do volume nasofaríngeo observado no repouso velar e na fala em 18 indivíduos com FVA e em 20 indivíduos com FVI são mostrados na Tabela 1. No grupo FVA, foram observados volumes médios de $23,2 \mathrm{~cm}^{3}$ no repouso velar e $15,9 \mathrm{~cm}^{3}$ durante a fala. A análise dos dados mostrou que esses valores diferiram significantemente entre si. No grupo FVI, os volumes médios corresponderam a $22,7 \mathrm{~cm}^{3}$ e $20,7 \mathrm{~cm}^{3}$, respectivamente, valores esses que, embora mais próximos, também diferiram estatisticamente entre si. A diferença entre o volume nasofaríngeo no repouso velar dos grupos FVA e FVI não foi estatisticamente significante, indicando dimensões nasofaríngeas equivalentes entre os grupos.

A Tabela 1 mostra, ainda, que no grupo FVA, a variação absoluta média do volume nasofaríngeo ( $\Delta \mathrm{VA})$ correspondeu a $-7,3 \mathrm{~cm}^{3}$ (denotando redução volumétrica), e que no grupo $\mathrm{FVI}$, foi de apenas $-2,0 \mathrm{~cm}^{3}$. A diferença entre os grupos foi estatisticamente significante. As variações relativas $(\Delta \mathrm{VR})$ corresponderam a $-31 \%$ e $-9 \%$, respectivamente, valores esses que também diferiram estatisticamente entre si.

A Figura 5 ilustra melhor a tendência observada de maiores variações do volume nasofaríngeo no grupo FVA, comparativamente ao que se verificou no grupo FVI, em especial, quando se analisa a diferença absoluta (em B) e a diferença relativa (em $\mathrm{C})$. 
Tabela 1 - Volumes nasofaríngeos: valores médios \pm desvio padrão, valores mínimos e máximos, diferença absoluta $(\triangle \mathrm{VA})$ e relativa $(\Delta \mathrm{VR})$, observados no repouso velar e na fala, em indivíduos com a função velofaríngea adequada (FVA) e inadequada (FVI).

\begin{tabular}{|c|c|c|c|c|}
\hline \multicolumn{5}{|c|}{ Volumes $\left(\mathrm{cm}^{3}\right)$} \\
\hline & $\begin{array}{l}\text { Repouso velar } \\
\left(V_{r}\right)\end{array}$ & $\begin{array}{l}\text { Fala } \\
\left(V_{k}\right)\end{array}$ & $\begin{array}{c}\Delta V_{A} \\
\left(V_{k}-V_{r}\right)\end{array}$ & $\begin{array}{c}\Delta V_{R} \% \\
\left(V_{k}-V_{r} / V_{r}\right)\end{array}$ \\
\hline FVA & $\begin{array}{c}23,2 \pm 3,6 \\
(14,6-35,1)\end{array}$ & $\begin{array}{l}15,9 \pm 3,8 \text { * } \\
(9,4-21,7)\end{array}$ & $-7,3$ & $-31 \%$ \\
\hline FVI & $\begin{array}{c}22,7 \pm 7,9 \\
(10,5-37,1)\end{array}$ & $\begin{array}{l}20,7 \pm 7,4^{*} \\
(6,5-34,2)\end{array}$ & $-2,0^{\#}$ & $-9 \%{ }^{\#}$ \\
\hline
\end{tabular}

${ }^{*} \mathrm{p}<0,05$ diferença estatisticamente significante (repouso velar $\mathrm{x}$ fala)

$\# p<0,05$ diferença estatisticamente significante (FVA $x$ FVI) 
(A)

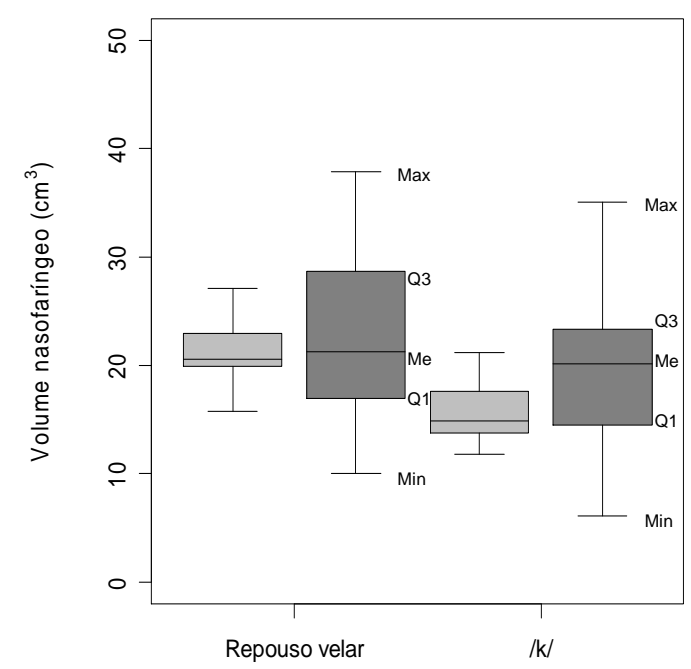

(C)

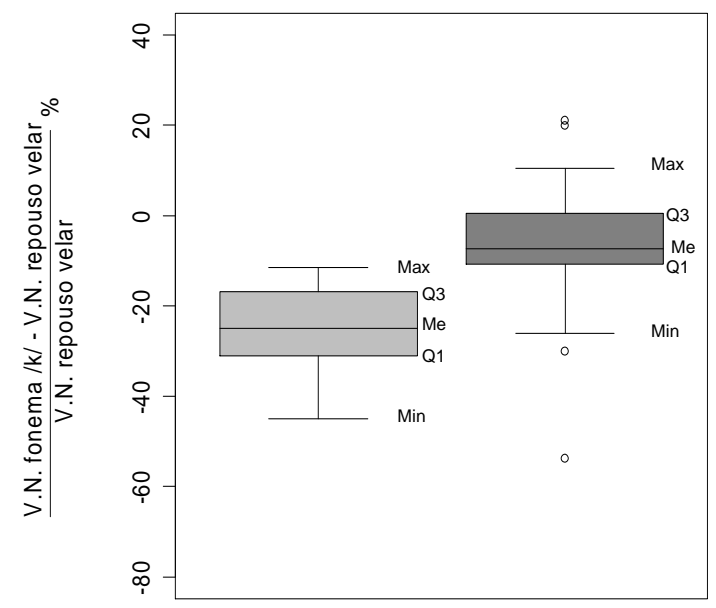

(B)

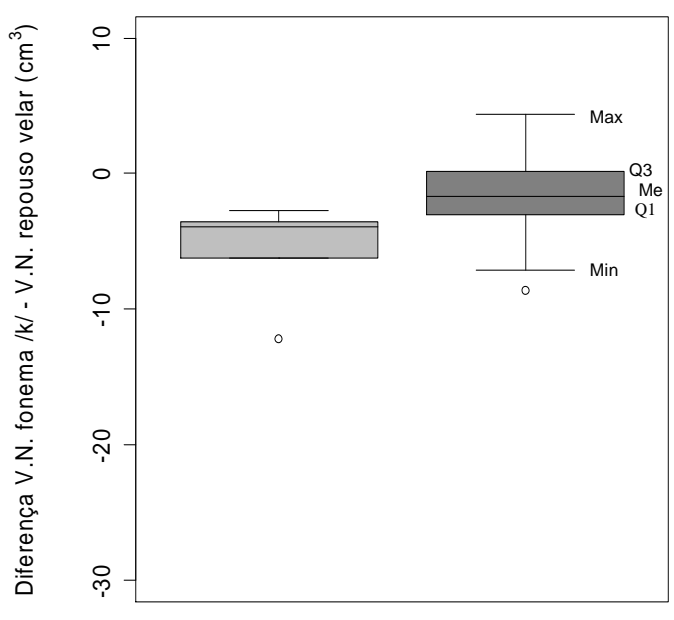

$\square$ FVA

FVI

Figura 5 - Comparação do volume nasofaríngeo observado nos indivíduos com função velofaríngea adequada (FVA) e inadequada (FVI). Em (A) são apresentados os volumes absolutos aferidos nos dois grupos, no repouso velar $(\mathrm{Vr})$ e na fala (produção do fonema /k/) (Vk). Em (B) são comparados as diferenças absolutas entre $\mathrm{Vr}$ e $\mathrm{Vk}$ e em (C) as diferenças relativas entre $\mathrm{Vr}$ e $\mathrm{Vk}$ nos dois grupos. Os traços horizontais representam o valor mediano do volume nasofaríngeo $(\mathrm{Me})$, o 1ํ e o $3^{\circ}$ quartis (Q1 e Q3) e os valores máximo (max) e mínimo (min) observados, desconsiderando os valores discrepantes (círculos) identificados conforme critério estabelecido por Tukey (1977). 


\subsection{ANÁLISE DOS VALORES INDIVIDUAIS DO VOLUME NASOFARÍNGEO NO REPOUSO VELAR E NA FALA}

Os valores individuais do volumes nasofaríngeo no repouso velar e na fala observados nos grupos FVA e FVI estão apresentados nas Tabelas 2 e 3, respectivamente, e as variações individuais entre o repouso e a fala, indicativas da mobilidade velar, são ilustradas nas Figuras 6a,b,c, 7a,b,c e na Figura 8.

Da análise da Tabela 2 e Figura 6a,b,c, observa-se que todos os indivíduos do grupo FVA apresentaram redução do volume nasofaríngeo, indicativa de elevação velar, característica da adequação do mecanismo velofaríngeo. Os valores

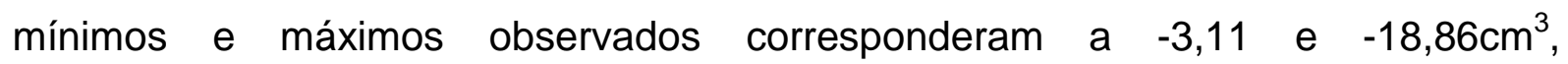
respectivamente.

Da análise da Tabela 3 e Figura 7a,b,c,d, observa-se que no grupo FVI, 9 pacientes não apresentaram redução significativa do volume nasofaríngeo durante a fala (menor que o valor de 3,0, a ser explicado mais adiante na Tabela 4). Este padrão, que sugere ausência de elevação velar, foi observado nos pacientes 5,8 , 10, 12, 14, 15, 17,19 e 20. Um número adicional de 5 pacientes demonstraram um aumento do volume nasofaríngeo, porém próximo ao valor de corte, não observado em nenhum indivíduo do grupo controle (pacientes 1, 6, 7, 13 e 18), também sugerindo ausência de elevação velar. Observa-se, por outro lado, que 6 pacientes apresentaram um decréscimo significativo do volume nasofaríngeo (maior que o valor de corte), comparável ao do grupo controle, sugerindo a presença de elevação velar apesar da IVF. Esse padrão foi observado nos pacientes 2, 3, 4, 9, 11 e 16. 
Tabela 2 - Volumes nasofaríngeos individuais: valores médios e diferença absoluta ( $\triangle \mathrm{VA})$ e relativa $(\Delta \mathrm{VR})$, observados no repouso velar e na fala, em indivíduos com a função velofaríngea adequada (FVA).

\begin{tabular}{ccccc}
\hline & & \multicolumn{2}{c}{ Volumes $\left(\mathbf{c m}^{\mathbf{3}}\right)$} & \\
\hline Pacientes & Repouso velar & Fala & $\Delta$ VA & $\Delta$ VR \\
\hline 1 & 35,1 & 16,2 & $-18,9$ & $-53,8 \%$ \\
\hline 2 & 29,2 & 21,7 & $-7,4$ & $-25,5 \%$ \\
\hline 3 & 24,8 & 16,9 & $-7,9$ & $-31,8 \%$ \\
\hline 5 & 19,4 & 13,3 & $-6,2$ & $-31,8 \%$ \\
\hline 6 & 21,2 & 13,8 & $-7,4$ & $-35,0 \%$ \\
\hline 7 & 26,6 & 19,8 & $-6,8$ & $-25,7 \%$ \\
\hline 8 & 15,7 & 11,9 & $-3,8$ & $-24,3 \%$ \\
\hline 9 & 20,0 & 13,3 & $-6,7$ & $-33,4 \%$ \\
\hline 10 & 35,1 & 18,0 & $-17,2$ & $-48,9 \%$ \\
\hline 11 & 21,7 & 17,6 & $-4,1$ & $-18,8 \%$ \\
\hline 12 & 17,1 & 12,9 & $-4,2$ & $-24,7 \%$ \\
\hline 13 & 21,9 & 18,8 & $-3,1$ & $-14,2 \%$ \\
\hline 14 & 16,9 & 13,0 & $-4,0$ & $-23,4 \%$ \\
\hline 15 & 27,5 & 20,1 & $-7,5$ & $-27,2 \%$ \\
\hline 16 & 14,6 & 9,4 & $-5,3$ & $-35,9 \%$ \\
\hline 17 & 25,5 & 19,4 & $-6,1$ & $-24,1 \%$ \\
\hline 18 & 15,7 & 9,9 & $-5,9$ & $-36,8 \%$ \\
\hline
\end{tabular}


Tabela 3 - Volumes nasofaríngeos individuais: valores médios e diferença absoluta ( $\triangle \mathrm{VA})$ e relativa $(\Delta \mathrm{VR})$, observados no repouso velar e na fala, em indivíduos com a função velofaríngea inadequada (FVI).

\begin{tabular}{|c|c|c|c|c|}
\hline \multirow[b]{2}{*}{ Pacientes } & \multicolumn{4}{|c|}{ Volumes $\left(\mathrm{cm}^{3}\right)$} \\
\hline & Repouso velar & Fala & $\Delta \mathbf{V A}_{\mathbf{A}}$ & $\Delta \mathbf{V}_{\mathbf{R}}$ \\
\hline 1 & 23,1 & 23,5 & $+0,3$ & $+1,4 \%$ \\
\hline 2 & 31,4 & 21,3 & $-10,1$ & $-32,0 \%$ \\
\hline 3 & 32,7 & 27,9 & $-4,8$ & $-14,7 \%$ \\
\hline 4 & 26,1 & 22,7 & $-3,4$ & $-13,2 \%$ \\
\hline 5 & 20,0 & 17,2 & $-2,8$ & $-13,9 \%$ \\
\hline 6 & 19,0 & 19,8 & $+0,9$ & $+4,6 \%$ \\
\hline 7 & 15,3 & 19,1 & $+3,8$ & $24,8 \%$ \\
\hline 8 & 10,6 & 9,9 & $-0,7$ & $-6,2 \%$ \\
\hline 9 & 15,5 & 10,6 & $-5,0$ & $-31,9 \%$ \\
\hline 10 & 18,0 & 15,1 & $-2,8$ & $-15,7 \%$ \\
\hline 11 & 37,1 & 32,1 & $-5,0$ & $-13,5 \%$ \\
\hline 12 & 24,7 & 23,0 & $-1,7$ & $-6,9 \%$ \\
\hline 13 & 10,5 & 12,4 & $+1,8$ & $+17,3 \%$ \\
\hline 14 & 24,2 & 23,0 & $-1,2$ & $-4,8 \%$ \\
\hline 15 & 36,7 & 34,2 & $-2,5$ & $-6,8 \%$ \\
\hline 16 & 13,7 & 6,5 & $-7,3$ & $-52,8 \%$ \\
\hline 17 & 29,9 & 29,5 & $-0,4$ & $-1,4 \%$ \\
\hline 18 & 22,9 & 25,6 & $+2,7$ & $+11,9 \%$ \\
\hline 19 & 19,2 & 18,6 & $-0,6$ & $-3,2$ \\
\hline 20 & 22,5 & 22,2 & $-0,3$ & $-1,4$ \\
\hline
\end{tabular}




\section{FVA}
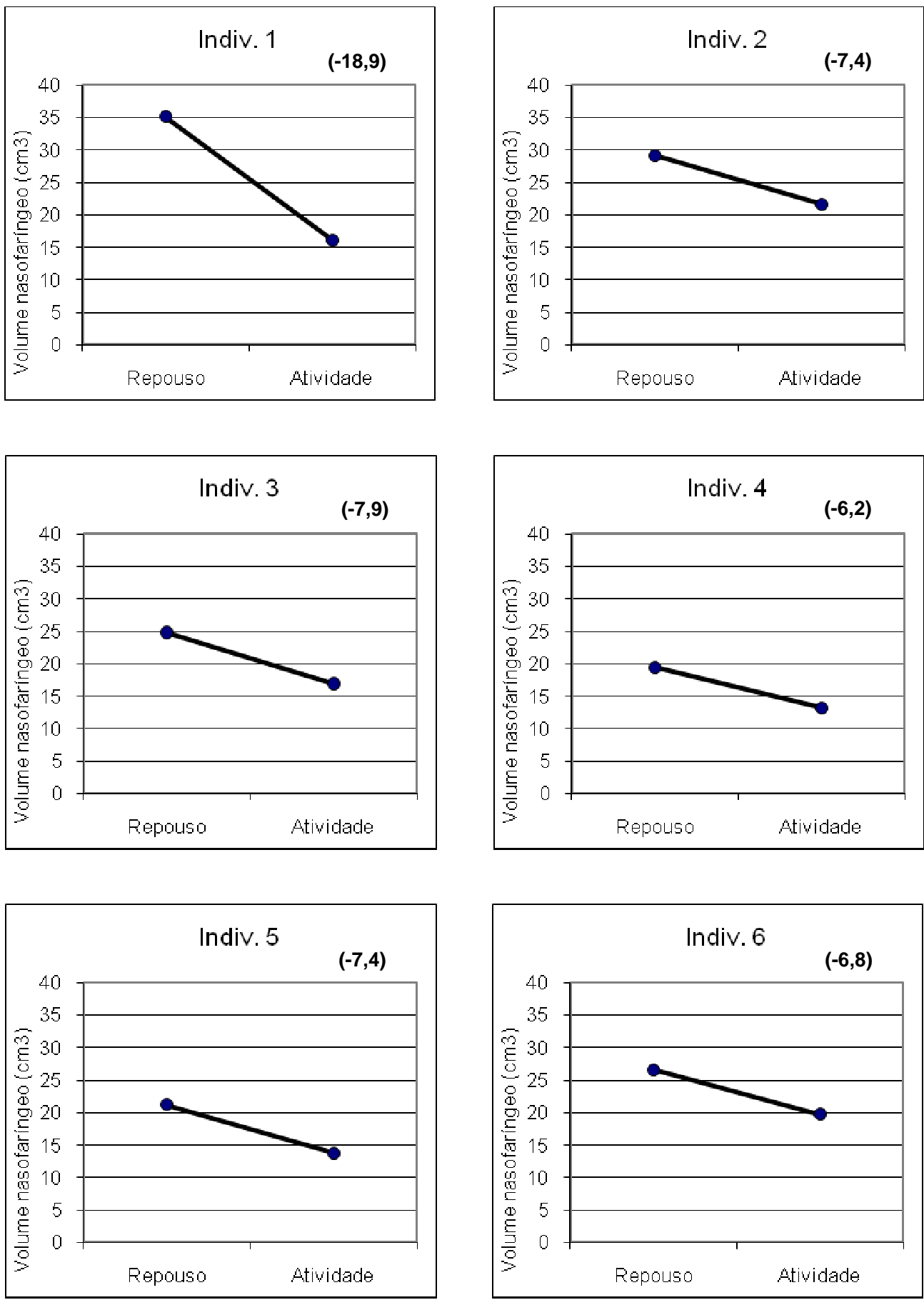

Figura 6a - Valores absolutos do volume nasofaríngeo no repouso velar e na fala observados nos indivíduos com função velofaríngea adequada (FVA). O número mostrado acima e à direita do traçado corresponde a variação volumétrica observada $(\Delta \mathrm{VA})$. 


\section{FVA}
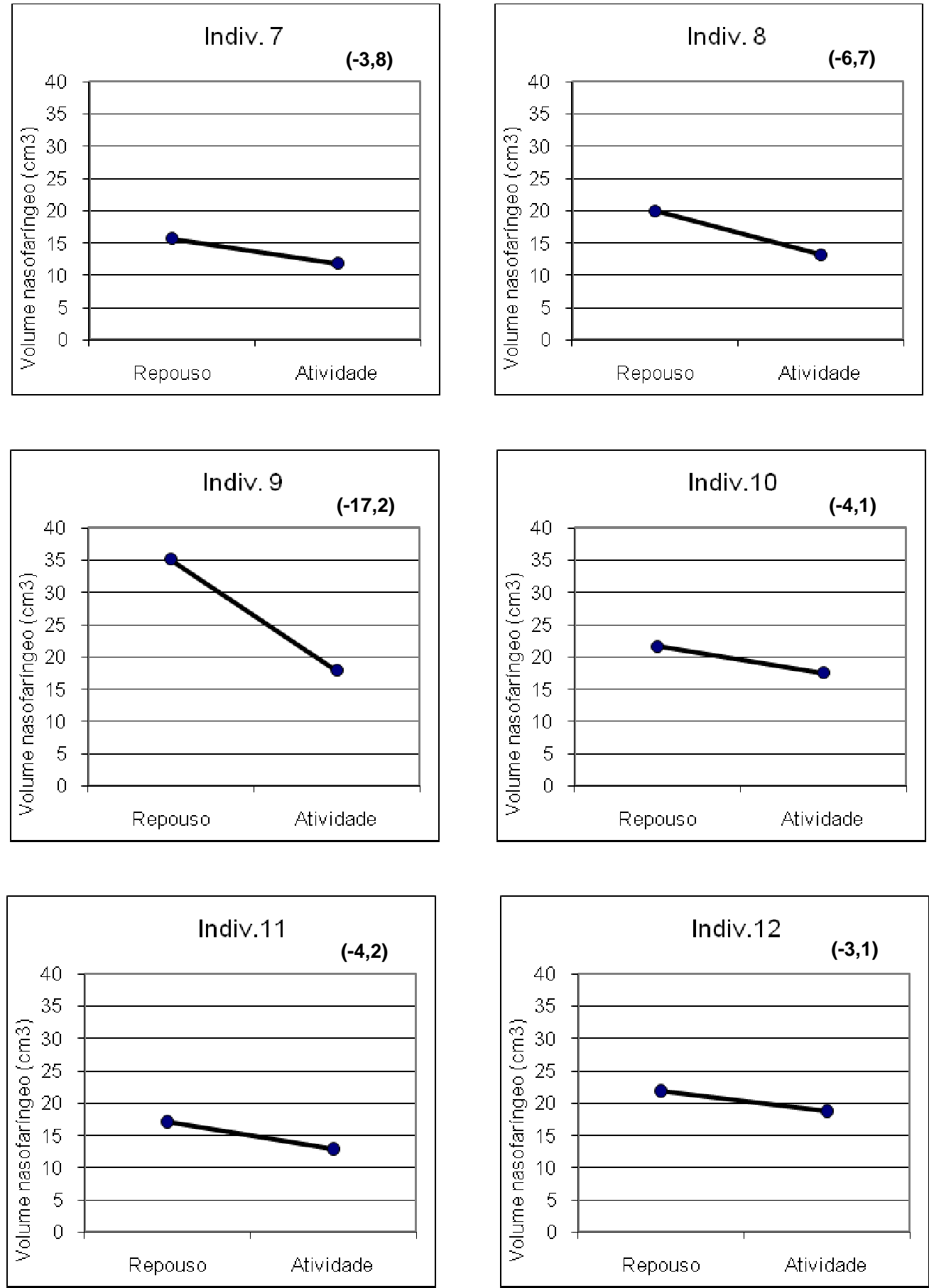

Figura 6b - Valores absolutos do volume nasofaríngeo no repouso velar e na fala observados nos indivíduos com função velofaríngea adequada (FVA). O número mostrado acima e à direita do traçado corresponde a variação volumétrica observada $(\Delta \mathrm{VA})$. 


\section{FVA}
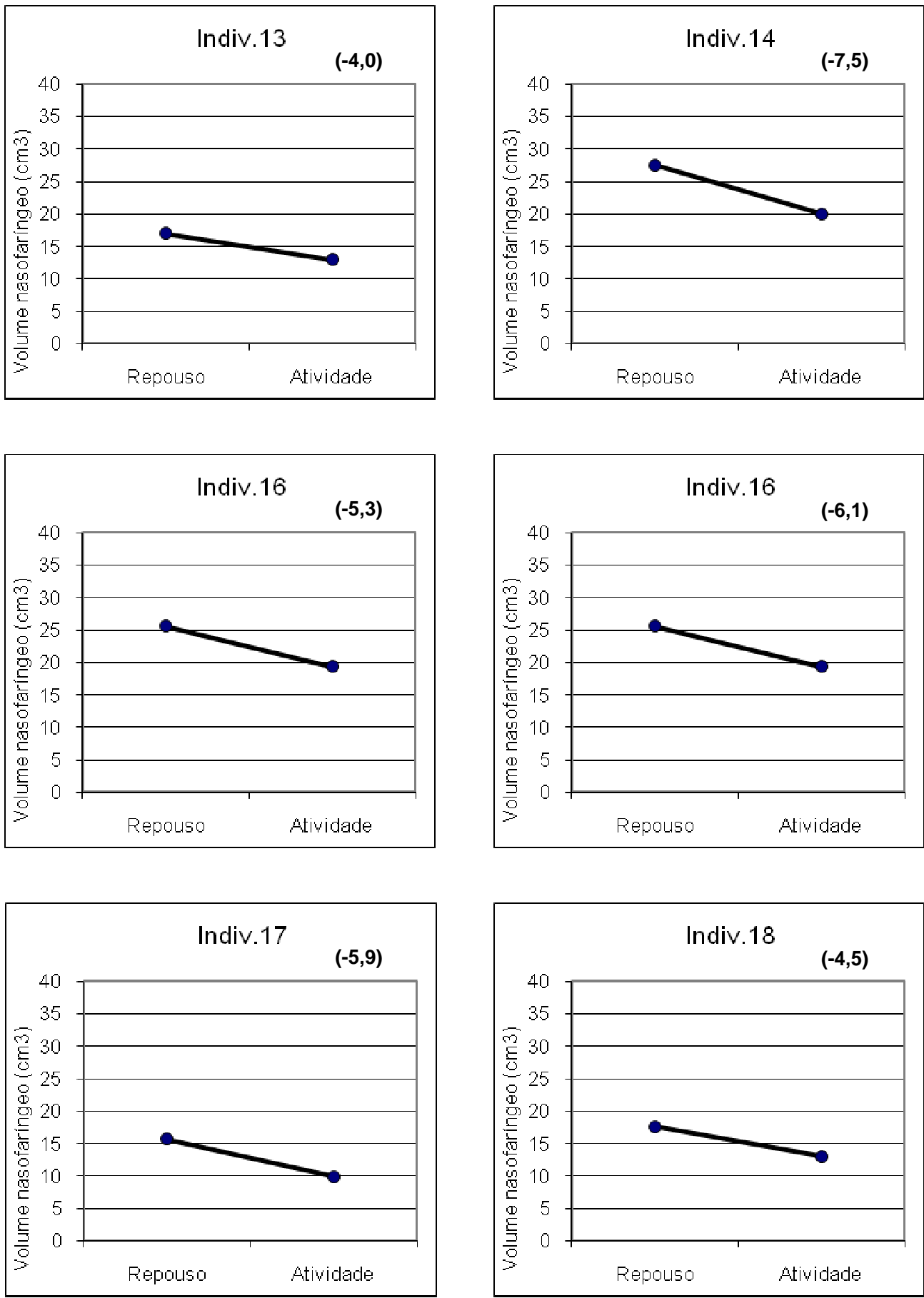

Figura 6c - Valores absolutos do volume nasofaríngeo no repouso velar e na fala observados nos indivíduos com função velofaríngea adequada (FVA). O número mostrado acima e à direita do traçado corresponde a variação volumétrica observada $(\Delta \mathrm{VA})$. 


\section{FVI}
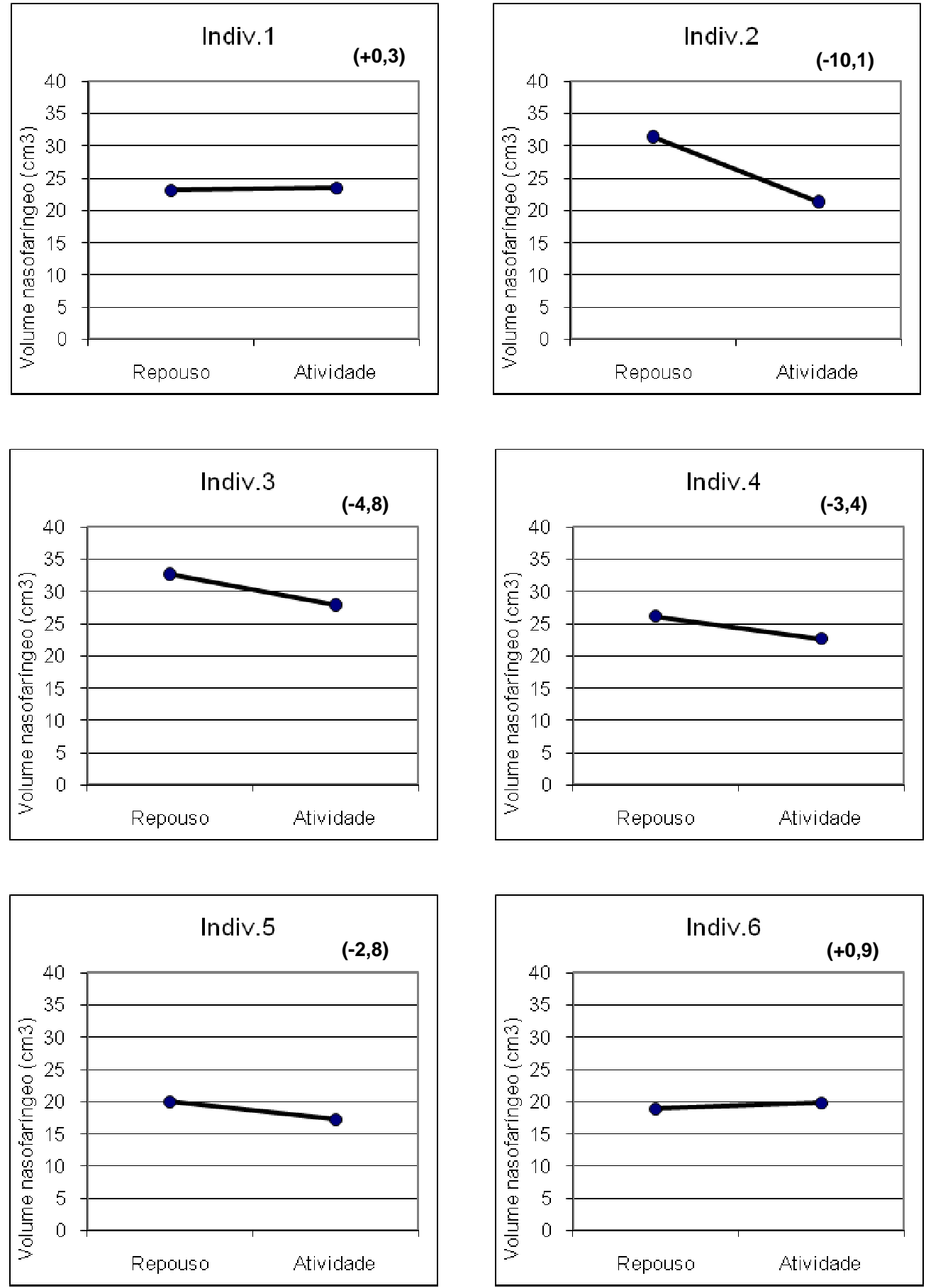

Figura 7a - Valores absolutos do volume nasofaríngeo no repouso velar e na fala observados nos indivíduos com função velofaríngea inadequada (FVI). Os indivíduos 1, 5 e 6 apresentaram resultado sugestivo de ausência de elevação velar. O número mostrado acima e à direita do traçado corresponde a variação volumétrica observada $(\Delta \mathrm{VA})$. 


\section{FVI}
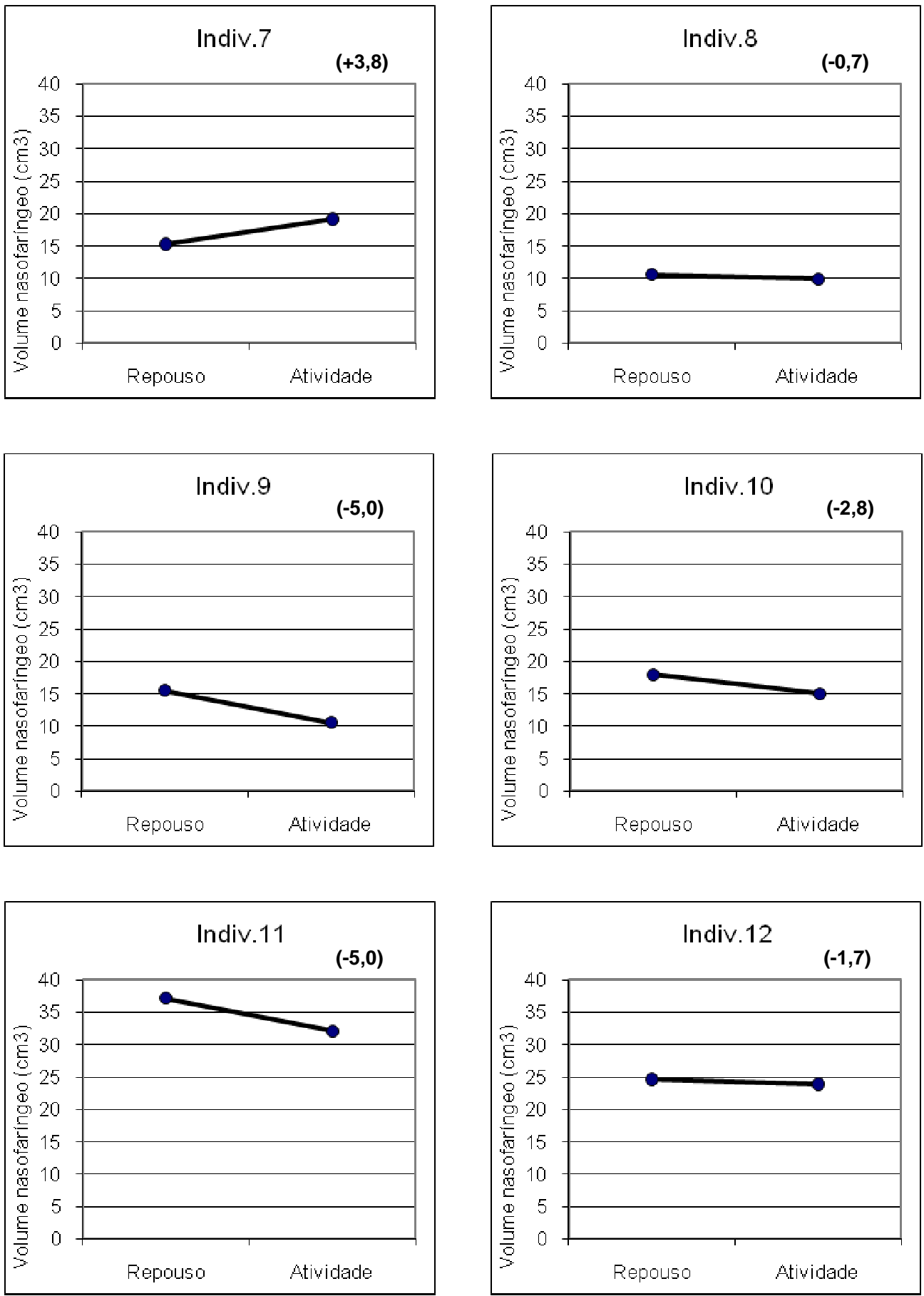

Figura 7b - Valores absolutos do volume nasofaríngeo no repouso velar e na fala observados nos indivíduos com função velofaríngea inadequada $(\mathrm{FVI})$. Os indivíduos 8, 10 e 12 apresentaram resultado sugestivo de ausência de elevação velar. O número mostrado acima e à direita do traçado corresponde a variação volumétrica observada $(\Delta \mathrm{VA})$. 


\section{FVI}
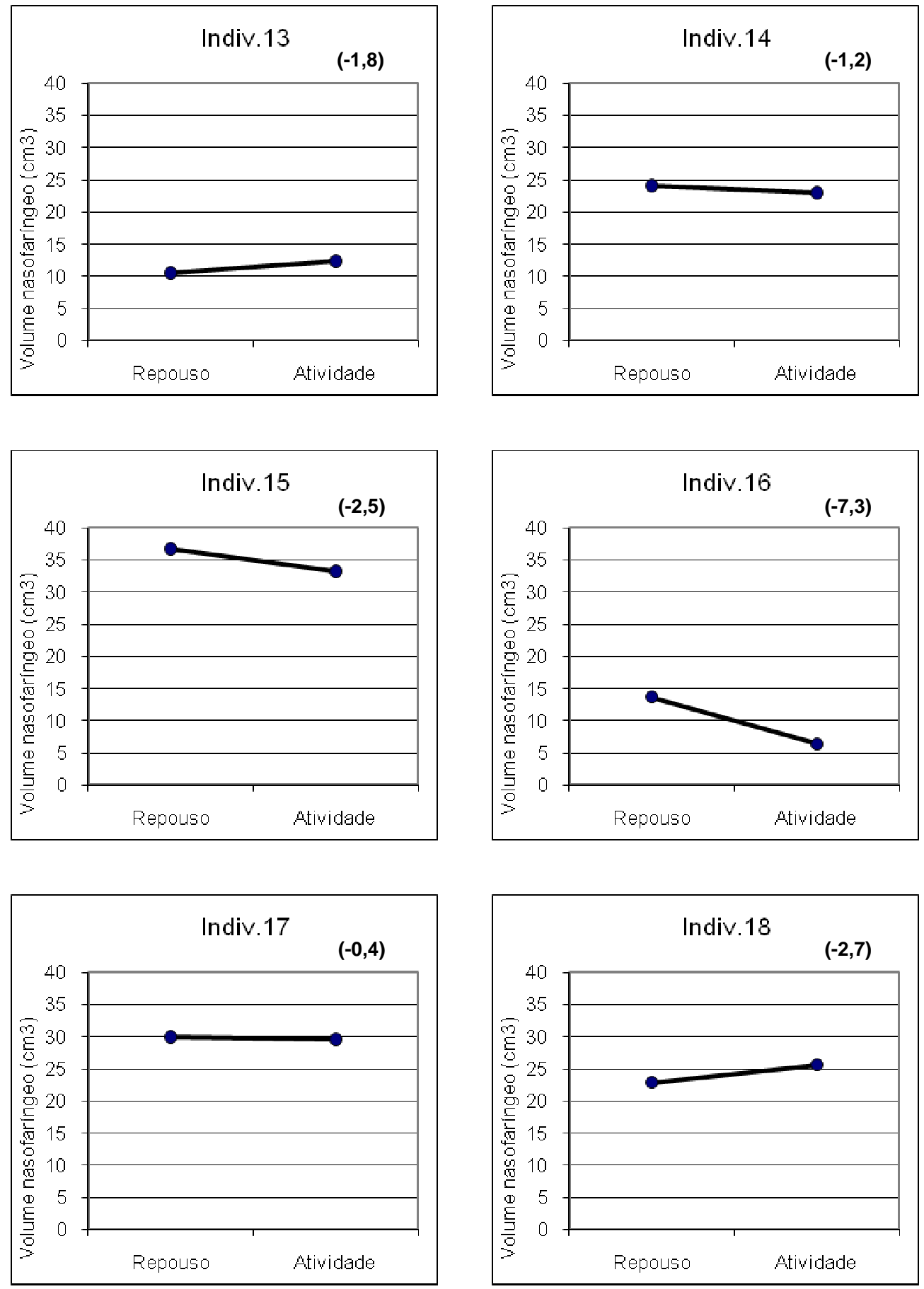

Figura 7c - Valores absolutos do volume nasofaríngeo no repouso velar e na fala observados nos indivíduos com função velofaríngea inadequada (FVI). Os indivíduos 13, 14, 15, 17 e 18 apresentaram resultado sugestivo de ausência de elevação velar. $O$ número mostrado acima e à direita do traçado corresponde a variação volumétrica observada $(\Delta \mathrm{VA})$. 
$\mathrm{FVI}$
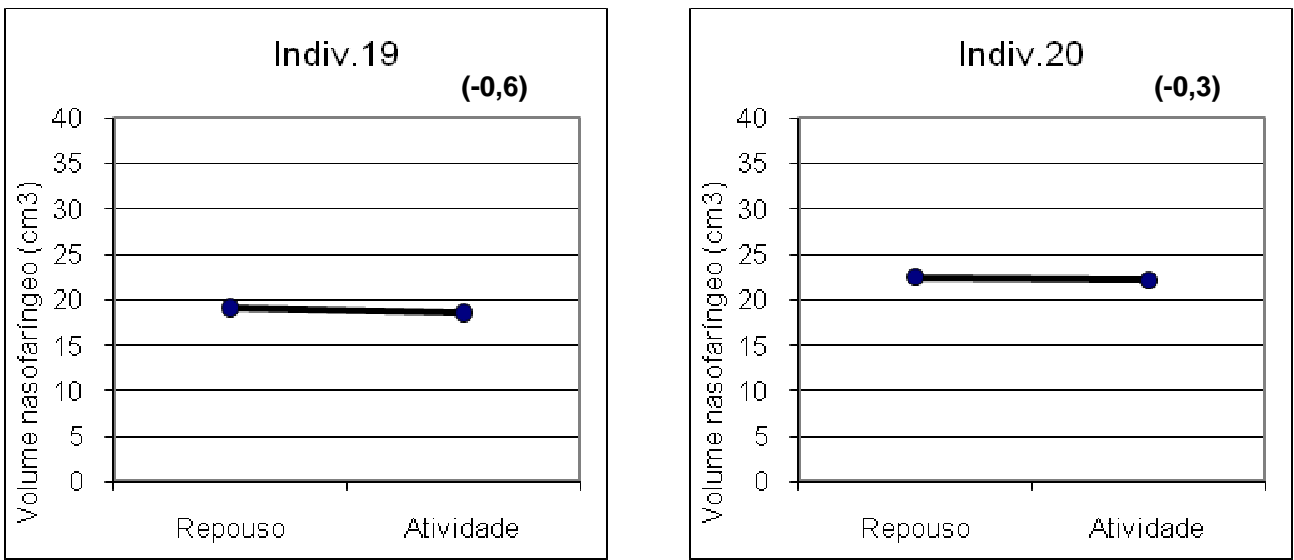

Figura 7d - Valores absolutos do volume nasofaríngeo no repouso velar e na fala observados nos indivíduos com função velofaríngea inadequada (FVI). Os indivíduos 19 e 20 apresentaram resultado sugestivo de ausência de elevação velar. O número mostrado acima e à direita do traçado corresponde a variação volumétrica observada $(\Delta \mathrm{VA})$. 


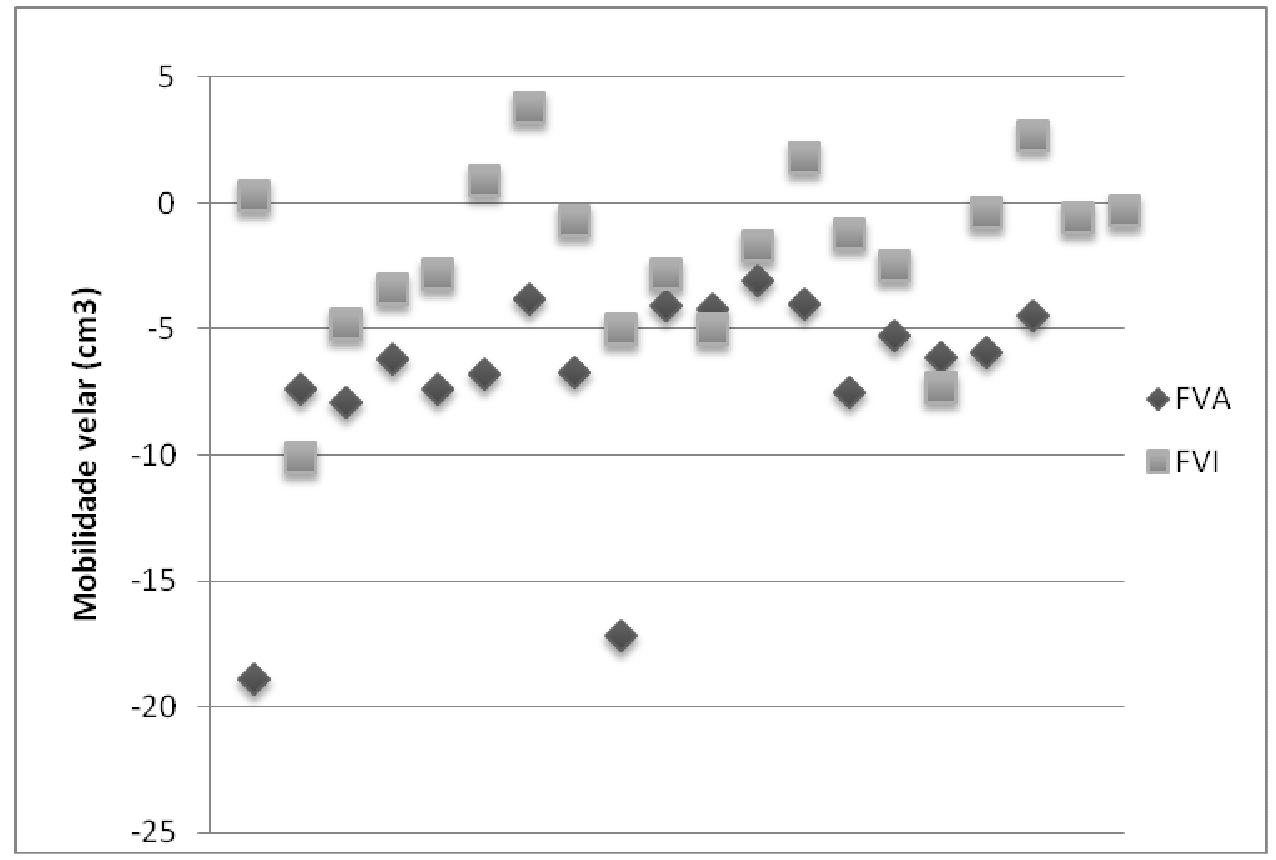

Figura 8 - Gráfico de dispersão ilustrando a mobilidade velar individual entre o grupo FVA e o grupo FVI. 


\subsection{ANÁLISE DA SENSIBILIDADE E ESPECIFICIDADE DA RINOMETRIA ACÚSTICA}

A determinação da sensibilidade e especificidade do teste, pela análise $\mathrm{ROC}$, para a definição do limite de normalidade de $\Delta \mathrm{V}$ (valor de corte), encontra-se nas Tabelas 4 e 5 . A Tabela 4 mostra que o valor de $\Delta \mathrm{VA}$ que maximizou simultaneamente a sensibilidade e a especificidade do teste correspondeu a -2,965. Isto significa que valores menores que $-3,0$ podem ser interpretados como indicativos de elevação velar inadequada. Para esse valor de corte, a sensibilidade e a especificidade corresponderam a 0,70 e 1,00, respectivamente, denotando que existe uma chance de $70 \%$ do teste identificar corretamente a deficiência da mobilidade velar e de $100 \%$ de identificar corretamente a adequação da mobilidade velar. Para o $\triangle \mathrm{VR}$, o valor de corte correspondeu a $-17 \%$ e a sensibilidade e a especificidade corresponderam a 0,85 e 0,94, respectivamente. 
Tabela 4 - Sensibilidade e especificidade da rinometria acústica como teste diagnóstico da disfunção velofaríngea para diferentes $\triangle \mathrm{VA}$ (diferença absoluta entre volume nasofaríngeo durante a produção do fonema $/ \mathrm{k} /$ e o repouso velar). $\mathrm{O}$ valor em negrito corresponde ao $\triangle \mathrm{VA}$ que maximizou a sensibilidade e a especificidade, simultaneamente, e, portanto, a ser considerado como valor de corte.

\begin{tabular}{|c|c|c|}
\hline$\Delta \mathrm{V}_{\mathrm{a}}$ & Sensibilidade & Especificidade \\
\hline$-19,860$ & 1,00 & 0,00 \\
\hline$-18,025$ & 1,00 & 0,06 \\
\hline$-13,625$ & 1,00 & 0,11 \\
\hline$-9,615$ & 0,95 & 0,11 \\
\hline$-8,525$ & 0,95 & 0,17 \\
\hline$-7,680$ & 0,95 & 0,22 \\
\hline$-7,455$ & 0,95 & 0,28 \\
\hline$-7,425$ & 0,95 & 0,33 \\
\hline$-7,335$ & 0,95 & 0,39 \\
\hline$-7,040$ & 0,90 & 0,39 \\
\hline$-6,750$ & 0,90 & 0,44 \\
\hline$-6,435$ & 0,90 & 0,50 \\
\hline$-6,170$ & 0,90 & 0,56 \\
\hline$-5,960$ & 0,90 & 0,61 \\
\hline$-5,515$ & 0,90 & 0,67 \\
\hline$-5,125$ & 0,90 & 0,72 \\
\hline$-4,980$ & 0,85 & 0,72 \\
\hline$-4,880$ & 0,80 & 0,72 \\
\hline$-4,660$ & 0,75 & 0,72 \\
\hline$-4,295$ & 0,75 & 0,78 \\
\hline$-4,015$ & 0,75 & 0,83 \\
\hline$-3,890$ & 0,75 & 0,89 \\
\hline$-3,630$ & 0,75 & 0,94 \\
\hline$-3,275$ & 0,70 & 0,94 \\
\hline$-2,965$ & 0,70 & 1,00 \\
\hline$-2,795$ & 0,65 & 1,00 \\
\hline$-2,635$ & 0,60 & 1,00 \\
\hline$-2,105$ & 0,55 & 1,00 \\
\hline$-1,430$ & 0,50 & 1,00 \\
\hline$-0,900$ & 0,45 & 1,00 \\
\hline$-0,635$ & 0,40 & 1,00 \\
\hline$-0,520$ & 0,35 & 1,00 \\
\hline$-0,370$ & 0,30 & 1,00 \\
\hline$-0,005$ & 0,25 & 1,00 \\
\hline 0,605 & 0,20 & 1,00 \\
\hline 1,350 & 0,15 & 1,00 \\
\hline 2,275 & 0,10 & 1,00 \\
\hline 3,265 & 0,05 & 1,00 \\
\hline 4,800 & 0,00 & 1,00 \\
\hline
\end{tabular}


Tabela 5 - Sensibilidade e especificidade da rinometria acústica como teste diagnóstico da disfunção velofaríngea para diferentes valores de $\Delta \mathrm{VR}$ (diferença relativa entre volume nasofaríngeo durante a produção do fonema $/ \mathrm{k} /$ e o repouso velar). $\mathrm{O}$ valor em negrito corresponde ao $\Delta$ VR que maximizou a sensibilidade e a especificidade, simultaneamente, e, portanto, a ser considerado como valor de corte.

\begin{tabular}{|c|c|c|}
\hline$\Delta \mathrm{V}_{\mathrm{r}}$ & Sensibilidade & Especificidade \\
\hline$-54,79$ & 1,00 & 0,00 \\
\hline$-53,30$ & 1,00 & 0,06 \\
\hline$-50,86$ & 0,95 & 0,06 \\
\hline$-42,88$ & 0,95 & 0,11 \\
\hline$-36,39$ & 0,95 & 0,17 \\
\hline$-35,48$ & 0,95 & 0,22 \\
\hline$-34,22$ & 0,95 & 0,28 \\
\hline$-32,71$ & 0,95 & 0,33 \\
\hline$-31,98$ & 0,90 & 0,33 \\
\hline$-31,89$ & 0,85 & 0,33 \\
\hline$-31,82$ & 0,85 & 0,39 \\
\hline$-31,45$ & 0,85 & 0,44 \\
\hline$-29,14$ & 0,85 & 0,50 \\
\hline$-26,43$ & 0,85 & 0,56 \\
\hline$-25,68$ & 0,85 & 0,61 \\
\hline$-25,56$ & 0,85 & 0,67 \\
\hline$-24,88$ & 0,85 & 0,72 \\
\hline$-24,18$ & 0,85 & 0,78 \\
\hline$-23,71$ & 0,85 & 0,83 \\
\hline$-21,09$ & 0,85 & 0,89 \\
\hline$-17,25$ & 0,85 & 0,94 \\
\hline$-15,19$ & 0,80 & 0,94 \\
\hline$-14,45$ & 0,75 & 0,94 \\
\hline$-14,04$ & 0,75 & 1,00 \\
\hline$-13,66$ & 0,70 & 1,00 \\
\hline$-13,32$ & 0,65 & 1,00 \\
\hline$-10,04$ & 0,60 & 1,00 \\
\hline$-6,86$ & 0,55 & 1,00 \\
\hline$-6,49$ & 0,50 & 1,00 \\
\hline$-5,46$ & 0,45 & 1,00 \\
\hline$-3,99$ & 0,40 & 1,00 \\
\hline$-2,32$ & 0,35 & 1,00 \\
\hline 1,41 & 0,30 & 1,00 \\
\hline$-0,01$ & 0,25 & 1,00 \\
\hline 3,04 & 0,20 & 1,00 \\
\hline 8,29 & 0,15 & 1,00 \\
\hline 14,60 & 0,10 & 1,00 \\
\hline 21,03 & 0,05 & 1,00 \\
\hline 25,79 & 0,00 & 1,00 \\
\hline
\end{tabular}






\section{DISCUSSÃO}

As variações do volume nasofaríngeo provocadas pela atividade velar, aferidas por rinometria acústica, foram o foco de atenção do presente estudo, o primeiro de uma série em desenvolvimento no Laboratório de Fisiologia do HRAC/USP para validar o uso da técnica como método de avaliação da mobilidade do véu do palato em atividades de fala. Como etapa inicial, definiu-se a magnitude da redução volumétrica da nasofaringe produzida por uma atividade de fala que exige máxima elevação velar - a produção do fonema //k/ - em indivíduos normais, e, a seguir, analisou-se o impacto da falta de tecido velar - a insuficiência velofaríngea sobre a magnitude da redução volumétrica da nasofaringe. Os resultados obtidos mostraram que a técnica foi capaz de discriminar parcela significativa de pacientes com função velofaríngea inadequada daqueles com função adequada e, ao mesmo tempo, de identificar um sub-grupo de pacientes com mobilidade velar preservada, apesar dos sintomas de IVF.

Para analisar o significado desses achados é preciso discutir inicialmente alguns aspectos metodológicos do estudo. Primeiramente, vale ressaltar que os resultados obtidos vão de encontro à proposta original de Dalston (1992) que atribuiu à rinometria acústica, em função de algumas características físicas do equipamento utilizado, a possibilidade de monitorar as modificações da atividade velofaríngea durante a fala. Contudo, como enfatizou o autor, esse tipo de avaliação tem de ser realizada durante o que chamou de "fala silenciosa", uma vez que nenhum som pode transitar pela cavidade sob investigação, exceto o gerado pelo próprio equipamento. 
Seaver et al (1995) desenvolveram um estudo que reforçou a hipótese de Dalston, utilizando como amostra de fala o fonema /f/ produzido de forma prolongada e sem ruído. Os autores analisaram a atividade velofaríngea de dois falantes normais por videofluoroscopia e rinometria acústica, realizadas simultaneamente, em duas condições: durante a suspensão da respiração (orifício velofaríngeo aberto), e durante a produção de um /f/ "silencioso" (orifício velofaríngeo fechado), assumindo que as diferenças entre as duas condições seriam resultantes da atividade velofaríngea. A mudança na posição velar foi monitorada pela comparação das imagens videofluoroscópicas obtidas no repouso e na fala, alinhadas entre si. $\mathrm{Na}$ rinometria acústica, a comparação foi feita, como no presente estudo, superpondo os rinogramas obtidos nas duas condições. A inspeção visual dos rinogramas indicou que as duas curvas área-distância se separavam a uma distância de aproximadamente 7 a $8 \mathrm{~cm}$ das narinas nos dois pacientes analisados, o que confirma dados obtidos no grupo controle do presente estudo, em que o ponto de divergência situou-se em $7,7 \mathrm{~cm}$ a partir das narinas. Vale destacar que esse ponto corresponde à região de transição entre o palato duro e o palato mole.

Seaver et al (1995) observaram, em adição, uma excelente correspondência entre os resultados de ambas as técnicas. Em um dos indivíduos, por exemplo, a videofluoroscopia demonstrou que o véu do palato se elevava acima do plano palatal a 7,4cm das narinas e que o ponto máximo de elevação velar ocorria a 9,2cm, valores estes muito próximos aos obtidos na rinometria acústica $(7,6$ e 9,5cm, respectivamente). Resultados similares foram observados no outro indivíduo avaliado.

Ainda que de caráter preliminar, o estudo de Seaver et al (1995) teve o mérito de ser o primeiro a validar os achados da rinometria acústica frente a 
informações anatômicas sobre o posicionamento velar na fala e o primeiro a operacionalizar a metodologia proposta por Dalston. E foi com base nesse estudo, que a presente investigação foi desenvolvida, exceto por uma diferença importante aqui se utilizou a produção do /k/, conforme feito por Kunkel et al (1998a,b). Esses autores não justificaram as razões do uso daquela amostra de fala, no lugar do /f/ originalmente proposto por Seaver et al (1995). No presente estudo, a opção pelo fonema / $\mathrm{k}$ baseou-se nos achados de um estudo preliminar (Araújo et al 2009) no qual se constatou que muitos pacientes com IVF apresentavam curvas instáveis durante produção prolongada do /f/. Atribuiu-se essa instabilidade à contaminação sonora causada pelo ruído gerado na passagem do ar expiratório pela cavidade oral, próprio do som fricativo, e por vezes impossível de ser evitado, associado ao escape nasal de ar, próprio da IVF. A partir dessa constatação passou-se a utilizar o fonema /k/ inserido na palavra /kasa/, na tentativa de uniformizar, o mais possível, o esforço respiratório, solicitando ao indivíduo examinado a prolongar a fase de imposição da pressão intra-oral do som plosivo até o término da aquisição dos dados. Os resultados obtidos no presente estudo vieram a comprovar que se trata de amostra de fala menos sujeita às instabilidades observadas para o som fricativo.

Com o propósito de validar a técnica, foram analisados dois grupos: um composto por indivíduos com função velofaríngea adequada (FVA), ou seja, sem IVF, e outro composto por indivíduos com função velofaríngea inadequada (FVI), ou seja, com IVF, segundo julgamento perceptivo-auditivo da fala, procedimento também adotado por Seaver et al (1995). Para tornar a avaliação mais objetiva e classificar a IVF utilizou-se o protocolo de avaliação da função velofaríngea proposto por Trindade et al (2005) que se baseia na atribuição de escores aos sinais associados à IVF (hipernasalidade, emissão de ar nasal e articulações 
compensatórias). Assim procedendo, apenas pacientes que apresentavam função velofaríngea inadequada (e não marginal) foram incluídos na presente investigação, além dos controles, com função velofaríngea adequada. Ressalte-se que avaliação rinométrica demonstrou que ambos os grupos apresentavam dimensões nasofaríngeas similares no repouso.

Ainda com relação a questões metodológicas, é preciso reenfatizar que a maior crítica feita na literatura à rinometria acústica (Terhryden et al 2000, Cakmak et al 2005) diz respeito exatamente a sua baixa acurácia em medir as dimensões das regiões mais posteriores da cavidade nasal, particularmente a nasofaringe, 0 que foi devidamente controlado no presente estudo. Isto porque se utilizou como medida de aferição da mobilidade velar o volume diferencial entre a condição de repouso e de atividade do véu palatino. Assim foi eliminada a interferência de fatores como a presença de obstruções significativas anteriores (lembrando também que a cavidade nasal de melhor permeabilidade foi sempre a escolhida para a realização das medidas) e a reflexão acústica para cavidade nasal contralateral e seios paranasais. Já, a ocorrência de movimentos palatinos involuntários e de variações do esforço respiratório, também apontadas na literatura como possíveis fatores intervenientes, foram variáveis não controladas; isto porque sua análise exigiria o uso de métodos complementares como a videofluoroscopia no primeiro caso, e a manometria oral no segundo, não previstos nesse primeiro estudo. Contudo, essa possibilidade foi minimizada pelas orientações dadas aos pacientes no sentido de evitá-las. Assim também foram tomadas todas as precauções no sentido de eliminar outros fatores que pudessem causar artefatos e interferir nas medidas realizadas, como detalhado por Gomes (2004). 
Isto posto, passemos à análise dos resultados encontrados fazendo uso da variação das dimensões da nasofaringe, na produção do fonema /k/ relativamente ao repouso $(\Delta \mathrm{V})$, como indicador da mobilidade velar na fala. Quando comparados os valores médios do $\Delta \mathrm{V}$ dos dois grupos analisados, o que se observou no grupo controle (FVA), foi uma redução significativa do volume nasofaríngeo de $7,3 \mathrm{~cm}^{3}$, correspondendo a uma variação relativa de 31\%. Gomes (2004), em estudo anterior do Laboratório, encontrou uma redução volumétrica da ordem de $20 \%$, em um grupo de indivíduos com função velofaríngea adequada. Porém, diferentemente do presente trabalho, em que os volumes da nasofaringe foram medidos num intervalo de $5 \mathrm{~cm}$ a partir do ponto de divergência das duas curvas analisadas (repouso e produção do fonema /k/), no trabalho de Gomes (2004), as medidas foram feitas durante a produção do fonema /f/, sempre no intervalo de 7 a $12 \mathrm{~cm}$ a partir da narina, tomando por base procedimento realizado com outras finalidades por Antila (1997). Já, Kunkel et al (1998a,b), usando a mesma metodologia empregada no presente estudo, exceto por analisarem a produção isolada do fonema /k/, relataram uma redução volumétrica de cerca de $8,0 \mathrm{~cm}^{3}$ nos pacientes com crescimento já completo, valor esse próximo ao encontrado no presente trabalho, o que valida a técnica empregada. Por outro lado, no grupo com função velofaríngea inadequada (FVI) observou-se uma redução volumétrica de apenas $2,0 \mathrm{~cm}^{3}$, não significativa, correspondendo a uma variação relativa de 9\%, significativamente menor que a observada no grupo FVA. Esse resultado é sugestivo de pouca mobilidade velar, compatível, portanto, com avaliação perceptiva da função velofaríngea.

Analisando mais uma vez os trabalhos de Kunkel et al (1998a,b), verifica-se que os autores obtiveram uma variação maior que a do presente estudo em indivíduos "com fissura" supostamente reparada cirurgicamente $\left(6,5 \mathrm{~cm}^{3}\right)$. Essa 
diferença decorre provavelmente das diferenças nas características da amostra de pacientes analisados por ambos os estudos. No presente trabalho, por exemplo, não foram incluídos indivíduos diagnosticados com fechamento velofaríngeo marginal, o que pode justificar, ao menos em parte, o menor valor encontrado. Ressalte-se que Kunkel et al (1998a,b) não fazem referência à presença de DVF no grupo analisado. Por outro lado, aqueles autores incluíram no estudo pacientes com retalho faríngeo, o que não se fez no presente estudo. Essa é uma questão que merece ser investigada em maiores detalhes. Por esta razão, a mobilidade velar de pacientes com função velofaríngea marginal já está sendo objeto de investigação de outro projeto em andamento no Laboratório de Fisiologia do HRAC/USP.

Em suma, a análise dos volumes médios amostrais permite concluir que a rinometria acústica foi capaz de discriminar indivíduos com e sem comprometimento da função velofaríngea. Contudo, a análise individual dos dados permite novas inferências. Assim procedendo, dois tipos de resposta foram observados durante a atividade de fala: em $70 \%$ dos casos de IVF analisados, ou seja, na maioria dos pacientes, comprovou-se a pouca mobilidade velar (pequenas variações volumétricas, para menos ou para mais, inferiores ao valor de corte de $3,0 \mathrm{~cm}^{3}$ ), inferida a partir da análise dos valores médios. Porém, contrariamente ao esperado, $30 \%$ dos casos apresentaram uma variação volumétrica comparável à do grupo controle (maior que o valor de corte), apesar de terem sido diagnosticados perceptivamente como tendo IVF. A superposição de casos e controles foi também observada por Kunkel et al (1998a,b), o que os levou a desconsiderar a possibilidade do uso do rinometria acústica como método para discriminar a função velofaríngea normal da patológica. Esta afirmação não parece procedente face às evidências dos resultados presente trabalho. Primeiro, porque no presente estudo, 
analisou-se uma amostra mais uniforme de pacientes e segundo porque a análise da sensibilidade e especificidade do método, ainda que de certa forma limitada pelo tamanho da amostra, mostrou-se elevada para o nível de corte de $3,0 \mathrm{~cm}^{3}(0,70$ e 1,00 , respectivamente).

Explicações para este achado encontram suporte em bases anatômicas e fisiológicas. É bem sabido que o mecanismo velofaríngeo compreende não só o movimento posterior e superior do palato mole, como também o movimento mesial das paredes laterais da faringe e, em menor grau, o movimento anterior da parede posterior da faringe (Moon e Kuehn 2004). Ocorre que a participação relativa desses componentes durante a fala varia de indivíduo para indivíduo (Shprintzen 1974, 1975, 1977). Com base nisto, reconhecem-se três tipos de fechamento: o coronal, o mais comum, quando predomina o movimento do palato mole, o circular, quando o palato mole e as paredes faríngeas contribuem igualmente para o fechamento, e, o sagital, o menos frequente, quando predomina o movimento mesial das paredes laterais da faringe (Kummer 2001), cuja extensão pode igualmente variar muito em falantes normais e anormais (Shprintzen et al 1977). A título de especulação, podese, talvez, atribuir o comportamento paradoxal observado ao exame rinométrico a uma falha na participação das paredes laterais da faringe no fechamento velofaríngeo, que explicaria a presença dos sintomas de IVF na presença de um bom movimento velar. Encontra-se em desenvolvimento no Laboratório de Fisiologia do HRAC/USP, um estudo no qual se pretende estabelecer a relação entre o grau de mobilidade velar aferido pela rinometria acústica e o tipo e grau de fechamento observado ao exame nasofaringoscópico.

Por outro lado, ao se aferir a variação volumétrica da nasofaringe na produção de um som plosivo, o que se avalia, em última análise, é a função dos 
músculos envolvidos no fechamento velofaríngeo - o levantador do véu do palato, o palatofaríngeo, o palatoglosso e o constritor superior da faringe, que agem através de interações sistemáticas e bastante complexas, como demonstrado por estudos eletromiográficos de Kuehn et al (1982). Essas interações variam na dependência da orientação e inserção dos músculos, o que é particularmente crítico em pacientes com fissura no palato, para os quais a elevação velar após a palatoplastia envolve mais esforço do que em normais (Kuehn e Moon 2004). Isto significa que a reconstituição cirúrgica do palato mole em pacientes com fissura congênita, como no caso dos pacientes do grupo experimental, ao mesmo tempo em que fornece as condições anatômicas para que o fechamento velofaríngeo se processe, pode não oferecer condições para que se sustente na fala habitual, por comprometimento do estado funcional dos músculos velofaríngeos, levando à persistência dos sintomas. Mais especificamente, isto pode ocorrer por falta de selamento completo e/ou persistente decorrente de deficiência na força de contato entre a superfície nasal do véu palatino e a parede posterior da faringe, ou por fadiga muscular, que explica 0 fechamento inconsistente por vezes observado (Moon e Kuehn 2004) ou por problemas de timing quando existe o fechamento, porém com certo retardo (Kummer 2001). Não se pode também excluir a possibilidade de erro de diagnóstico no julgamento perceptivo da fala, o que parece pouco provável em vista da longa experiência do fonoaudiólogo responsável pelas avaliações do presente estudo. O uso de um método objetivo de avaliação do grau de fechamento velofaríngeo, como a técnica fluxo-pressão de Warren (Trindade et al 2007), para dirimir esta questão, será objeto de estudo em um próximo trabalho do Laboratório de Fisiologia do HRAC/USP. 
O conjunto de dados mostra, enfim, que a rinometria acústica pode ser incorporada ao arsenal de métodos de análise da função velofaríngea diretos (nasofaringoscopia e videofluoroscopia) e indiretos (nasometria e técnica fluxopressão), para o que contribui o fato de ser de fácil execução, rápida, não invasiva e bem tolerada pelo paciente. Ainda que não definitivamente demonstrada sua eficácia no diagnóstico da disfunção velofaríngea, certamente parece ser um método útil para acompanhar intervenções cirúrgicas e terapêuticas que levem a melhor mobilidade velar, tema que também está sendo presentemente investigado no Laboratório de Fisiologia do HRAC/USP. 




\section{CONCLUSÕES}

No presente estudo foram analisadas as variações do volume da nasofaringe na produção de um som plosivo da fala, relativamente ao repouso, por meio de rinometria acústica, em indivíduos com fissura de palato reparada e diagnóstico de função velofaríngea inadequada e em indivíduos controles sem fissura e função velofaríngea adequada.

Os resultados obtidos mostraram que:

1) Indivíduos dos dois grupos apresentavam dimensões da nasofaringe similares;

2) A redução volumétrica média causada pela atividade velar durante a fala foi significantemente menor no grupo com função velofaríngea inadequada $(9 \%)$ que no grupo controle $(31 \%)$, indicando comprometimento da mobilidade velar no primeiro grupo, e, confirmando, dessa forma, o diagnóstico perceptivo;

3) A análise ROC permitiu identificar como limiar de normalidade ou valor de corte, uma variação volumétrica de $3,0 \mathrm{~cm}^{3}$, indicando que variações inferiores a esse valor devem ser consideradas como indicativas de anormalidade da função velar ou velofaríngea;

4) Com base nesse valor de corte, $70 \%$ dos pacientes do grupo com função inadequada apresentaram resultado sugestivo de comprometimento da elevação velar, refletindo o resultado médio observado. Os demais apresentaram resultado sugestivo de boa mobilidade velar, contrariando diagnóstico perceptivo, com significado ainda por esclarecer. 
Esses achados demonstram que a rinometria acústica foi capaz de identificar, com bom poder discriminatório, a deficiência do movimento velar na fala de indivíduos com diagnóstico clínico de insuficiência velofaríngea, o que se mostra particularmente útil para o acompanhamento de intervenções cirúrgico-terapêuticas.

O estudo contribui, dessa forma, para a validação da técnica como método de avaliação da atividade velofaríngea. 
7 REFERÊNCIAS 



\section{REFERÊNCIAS}

Antila J, Sipilä J, Tshushima Y, Pólo O, Laurikainen E, Suonpää. The effect of laseruvulopalatopharyngoplasty on the nasal and nasopharyngeal volume measured with acoustic rhinometry. Acta Otolaryngol Suppl 1997; 529:202-5.

Araújo BMAM, Sampaio-Teixeira ACM, Trindade IEK. Rinometria acústica: eficiência de diferentes fonemas na identificação da disfunção velofaríngea. In: Anais do Simpósio Internacional de Fissuras Orofaciais e Anomalias Relacionadas e $7^{\mathbf{0}}$ Encontro Científico de Pós-Graduação do HRAC-USP; 2009 Nov. 27-28; Bauru, Brasil. Bauru: Hospital de Reabilitação de Anomalias Craniofaciais, Universidade de São Paulo; 2009.

Bertier CE, Trindade IEK. Deformidades nasais: avaliação e tratamento cirúrgico. In: Trindade IEK, Silva Filho OG, org. Fissuras labiopalatinas: uma abordagem interdisciplinar. São Paulo: Santos; 2007. p.87-107.

Cakmak O, Tarhan E, Coskun M, Cankurtaran M, Celik H. Acoustic rhinometry: accuracy and ability to detect changes in passage area at different location in the nasal cavity. Ann Otol Rhinol Laryngol 2005;114:949-57.

Carvalho ELL. Resultado em pacientes submetidos à palatoplastia secundária associada à veloplastia intravelar [tese]. Bauru: Hospital de Reabilitação das Anomalias Craniofaciais, Universidade de São Paulo; 2006.

Clement PA, Gordts F. Consensus report on acoustic rhinometry and rhinomanometry. Rhinolgy 2005;43:169-79.

Corey J, Gungor A, Nelson R, Liu X, Fredberg J. Normative Standards for nasal cross-sectional areas by race as measured by acoustic rhinometry. Otolaryngeal Head Neck Surg 1998;119:389-93.

Couniham DT, Cullinam WL. Reability and dispersion of nasality ratings. Cleft Palate J 1970;7:261-70.

Dalston RM, Marsh JL, Vig KW, Witzel MA, Bumsted RM. Minimal standards for reporting the results of surgery on patients with cleft lip, cleft palate, or both: a proposal. Cleft Palate Craniofac J 1988;25:3-7.

Dalston RM. Acoustic assessment of the nasal airway. Cleft Palate Craniofac $J$ 1992;29:520-6. 
Dalston RM. The use of nasometry in the assessment and remediation of velopharyngeal inadequacy. In: Bzoch KR, editor. Communicative disorders related to cleft lip and palate. $5^{\text {th }}$ ed. Austin: Pro-ed; 2004. p.493-516.

Fisher EW, Morris DP, Biemans JMA, Palmer CR, Lund VJ. Pratical aspects of acoustic rhinometry: problems and solutions. Rhinology 1995;33:219-23.

Fukushiro AP. Análise perceptiva, nasométrica e aerodinâmica da fala de indivíduos submetidos à cirurgia de retalho faríngeo para a correção da insuficiência velofaríngea [tese]. Bauru: Hospital de Reabilitação de Anomalias Craniofaciais, Universidade de São Paulo; 2007.

Genaro KF, Yamashita RP, Trindade IEK. Avaliação clínica e instrumental na fissura labiopalatina. In: Ferreira LP, Befi-Lopes DM, Limongi SCO, org. Tratado de fonoaudiologia. São Paulo: Roca; 2004. p.456-77.

Genaro KF, Fukushiro AP, Suguimoto MLFCP. Avaliação dos distúrbios da fala. In: Trindade IEK, Silva Filho OG, org. Fissuras labiopalatinas: uma abordagem interdisciplinar. São Paulo: Santos; 2007. p.109-22.

Golding-Kushner KJ. Treatment of articulation and resonance disorders associated with cleft palate and VPI. In: Shprintzen RJ, Barbach J, editors. Cleft palate speech management: a multidisciplinary approach. St. Louis: Mosby; 1995. p.327-51.

Gomes AOC. Dimensões nasais e nasofaríngeas de indivíduos sem evidências de obstrução nasal avaliadas por rinometria acústica no repouso e na fala [dissertação]. Bauru: Hospital de Reabilitação das Anomalias Craniofaciais, Universidade de São Paulo; 2004.

Gomes AOC. Dimensões internas nasais de crianças com fissura de lábio e palato unilateral aferidas por rinometria acústica [tese]. Bauru: Hospital de Reabilitação de Anomalias Craniofaciais, Universidade de São Paulo; 2007.

Gomes AOC, Sampaio-Teixeira ACM, Trindade SHK, Trindade IEK. Áreas seccionais de adultos sadios aferidas por rinometria acústica. Rev Bras Otorrinolaringol 2008;74:746-59.

Hilberg O, Jackson AC, Swift DL, Pedersen OF. Acoustic rhinometry: evaluation of nasal cavity geometry by acoustic reflection. J Appl Physiol 1989;66:295-303.

Hilberg O, Jensen FT, Pedersen OF. Nasal airway geometry: comparison between acoustic reflections and magnetic resonance scanning. J Appl Phisiol 1993;75:281119. 
Hilberg O, Pedersen OF. Acoustic rhinometry: recommendations for technical specifications and standard operating procedures. Rhinol Supp/ 2000;16:3-17.

Hilberg O. Objective measurement of nasal airway dimensions using acoustic rhinometry: methodological and clinical aspects. Allergy 2002;57:5-39.

Jackson AC, Butler JP, Millet EJ, Hoppin Junior FG, Dawson SV. Airway geometry by analysis of acoustic pulse response measurements. J Appl Phisiol 1977;43:523-26.

Kuehn D, Folkins J, \& Cutting C. Relationships between muscle activity and velar position. Cleft Palate Craniofac J 1982;19:25-35.

Kummer AW. Cleft Palate and Craniofacial anomalies: the effects on speech and resonace. San Diego: Singular; 2001. 481p.

Kunkel M, Hochban W. Acoustic rhinometry: a new diagnostic procedureexperimental and clinical experience. Int J Oral Maxillofac Surg 1994a;23:409-12.

Kunkel M, Hochban W. Acoustic rhinometry: rationale and perspectives. J Cranio Max Fac Surg 1994b;22:244-9.

Kunkel M, Wahlmann $\mathrm{U}$, Wilfried W. Objective, noninvasive, evaluation of velopharyngeal function in cleft and noncleft patients. Cleft Palate Craniofac $J$ 1998a;35:35-39.

Kunkel M, Wahlmann U, Wagner W. Objective evaluation of velopharyngeal function by acoustic reflection measurements. Mund Kiefer Gesichtschir 1998b;2:158-62.

Moon JB, Kuehn DP. Anatomy and physiology of normal and disorded velopharyngeal function for speech. In: Bzoch KR, editor. Communicative disorders related to cleft lip and palate. $5^{\text {th }}$ ed. Austin: Pro-ed; 2004. p.67-98.

Park S H, Goo J M, Jo C. Receiver operating characteristic (ROC) curve: pratical review for radiologists. Korean J Radiol 2004;5:11-18.

Peterson-Falzone SJ, Hardin-Jones MA, Karnell MP. Communication disorders associated with cleft palate. In: Peterson-Falzone SJ, Hardon-Jones MA,Karnell MP. Cleft palate speech. St. Louis: Mosby; 2001. p.162-98.

Prado PC. Dimensões internas nasais de adultos com obstrução nasal avaliadas por rinometria acústica [dissertação]. Bauru: Hospital de Reabilitação das Anomalias Craniofaciais, Universidade de São Paulo; 2009. 
Rocha DL. Tratamento cirúrgico da insuficiência velofaríngea. In: Trindade IEK, Silva Filho OG, org. Fissuras labiopalatinas: uma abordagem interdisciplinar. São Paulo: Santos; 2007. p.145-63.

Roithmann R, Cole P. Objective assessment of nasal patency: why, when, how? Rev Bras Otorrinolaringol 1995;61:104-9.

Seaver EJ, Karnell MP, Gasparaitis A, Corey J. Acosutic rhinometric measurements of changes in velar posittioning. Cleft Palate Craniofac J 1995;32:49-54.

Shprintzen RJ, Lenicone RM, McCall GN, Skolnick ML. A three dimensional cinefluoroscopic analysis of velopharyngeal closure during speech and nonspeech activities in normals. Cleft Palate Craniofac J 1974;11:412-28.

Shprintzen RJ, McCall GN, Skolnick ML, Lenicone RM. Selective movement of the lateral aspects of the pharyngeal walls during velopharyngeal closure for speech, blowing, and whistling in normals. Cleft Palate Craniofac J 1975;12:51-8.

Shprintzen RJ, Rakof SJ, Skolnick ML, Lavorato AS. Incongruous movements of the velum and lateral pharyngeal walls. Cleft Palate Craniofac J 1977;14:148-57.

Silva Filho OG, Freitas JAS. Caracterização morfológica e origem embriológica. In: Trindade IEK, Silva Filho OG, org. Fissuras labiopalatinas: uma abordagem interdisciplinar. São Paulo: Santos; 2007. p.17-49.

Sondhi MM, Gopinath B. Determination of vocal tract shape from impulse response at the lips. J Acoustic Soc Am 1971;49:1867-73.

Terheyden $\mathrm{H}$, Maune S, Mertens J, Hilberg O. Acoustic rhinometry: validation by three-dimensionally reconstructed computer tomographic sacans. J Appl Phisiol 2000;89:1013-1021.

Trindade IEK, Genaro KF, Yamashita RP, Miguel HC, Fukushiro AP. Proposta de classificação da função velofaríngea na avaliação perceptivo-auditiva da fala. ProFono 2005;17:259-62.

Trindade IEK, Yamashita RP, Gonçalves CGAB. Diagnóstico instrumental da disfunção velofaríngea. In: Trindade IEK, Silva Filho OG, org. Fissuras labiopalatinas: uma abordagem interdisciplinar. São Paulo: Santos; 2007a. p.123-43.

Trindade IEK, Gomes AOC, Sampaio-Teixeira ACM, Trindade SHK. Volumes nasais de adultos aferidos por rinometria acústica. Rev Bras Otorrinolaringol 2007b;73:32-9. 
Trindade IE, Bertier CE, Sampaio-Teixeira AC. Objective assessment of internal nasal dimensions and speech resonance in individuals with repaired unilalateral cleft lip and palate after rhinoseptoplasty. J Craniofac Surg 2009;20:308-14.

Trindade IE, Castilho RL, Sampaio-Teixeira AC, Trindade-Suedam IK, Silva-Filho OG. Effects of orthopedic rapid maxillary expansion on internal nasal dimensions in children with cleft lip and palate assessed by acoustic rhinometry. J Craniofac Surg 2010;21:306-11.

Trost-Cardamone JE. Coming to terms with VPI: a reponse to Loney and Bloem. Cleft Palate Craniofac J 1989;26:68-70.

Trost-Cardamone JE. Diagnosis of specific cleft palate speech error patterns for planning therapy or physical management needs. In Bzoch KR,editor. Communicative disorders related to cleft lip and palate. $5^{\text {th }}$ ed. Austin: Pro-ed; 2004. p.463-91.

Tukey JW. Exploratory data analysis. Reading: Addison-Wesley; 1977.

Verbeke G, Molenberghs G. Linear mixed models in pratice. New York: Springer Verlag. 306p.

Vig OS, Spalding PM, Lints RR. Sensitivity and specificity of diagnostic tests for impaired nasal respiration. Am J Orthod Dentofacial Orthop 1991;99:354-60. 




\section{ANEXO 1 - OFÍCIO DE APROVAÇÃO DO PROJETO EMITIDO PELO COMITÊ DE ÉTICA EM PESQUISA EM SERES HUMANOS}

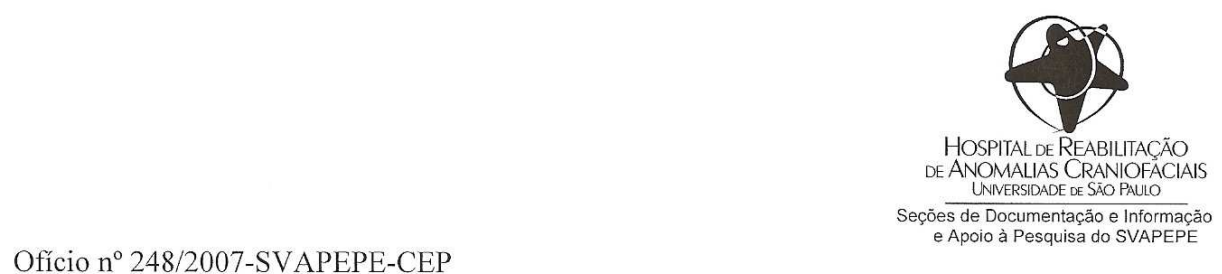

Bauru, 19 de novembro de 2007.

Prezado(a) Senhor(a)

O projeto de pesquisa encaminhado a este Comitê de Ética em Pesquisa em Seres Humanos, denominado "Avaliação da mobilidade velar em indivíduos com insuficiência velofaríngea por rinometria acústica.", de autoria de BRUNA MARA ADORNO MARMONTEL ARAÚJO desenvolvido sob sua orientação, foi enviado ao relator para avaliação.

$\mathrm{Na}$ reunião de $\mathbf{3 1 / 1 0 / 2 0 0 7}$ o parecer do relator, aprovando o projeto, foi aceito pelo Comitê, considerando que não existem infrações éticas pendentes para início da pesquisa. Solicitamos a V.S a gentileza de comunicar o parecer à pesquisadora e anexar o presente ofício ao projeto, pois o mesmo será necessário para futura publicação do trabalho.

A pesquisadora fica responsável pela entrega no SVAPEPE - Apoio ao Projeto de Pesquisa dos relatórios semestrais, bem como comunicar ao CEP todas as alterações que possam ocorrer no projeto.

Informamos que após o recebimento do trabalho concluído, este Comitê enviará o parecer final para publicação.

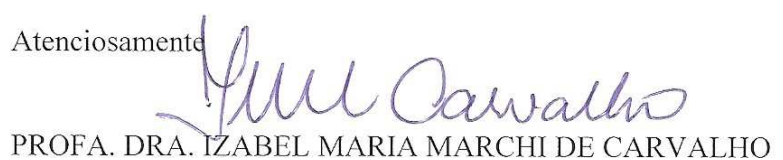

Coordenadora do Comitê de Ética em Pesquisa em Seres Humanos do HRAC-USP

$\operatorname{Ilmo}(\mathrm{a}) \operatorname{Sr}(\mathrm{a})$

Profa. Dra. Inge Elly Kiemle Trindade

Fisiologia - HRAC/USP

Rua Silvio Marchione, 3-20 Bauru SP Brasil Caixa Postal 1501 CEP 17.012-900

Tel. 551432358421

E-mail: cep@centrinho.usp.br 
ANEXO 2 - TERMO DE CONSENTIMENTO LIVRE E ESCLARECIDO

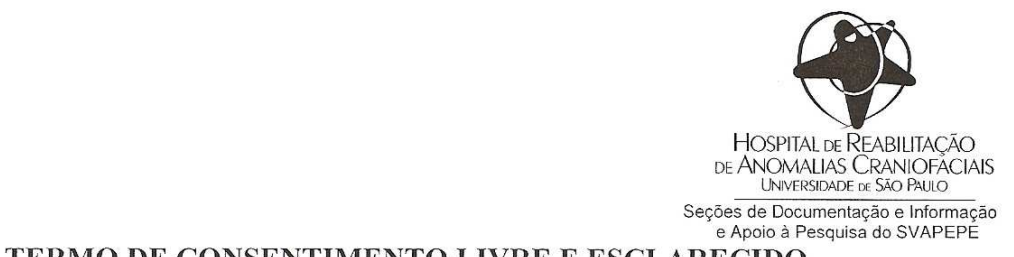

TERMO DE CONSENTIMENTO LIVRE E ESCLARECIDO

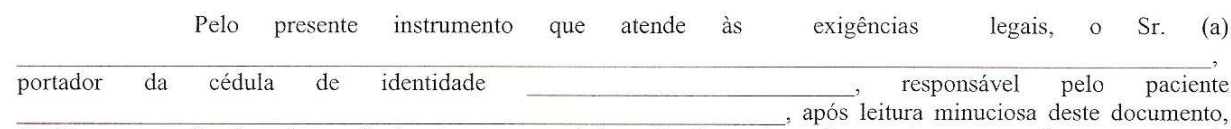

devidamente explicado pelos profissionais em seus mínimos detalhes, ciente dos serviços e procedimentos aos quais será submetido, não restando quaisquer dúvidas a respeito do lido e explicado, firma seu CONSENTIMENTO LIVRE E ESCLARECIDO concordando $\mathrm{em}$ participar da pesquisa: Avaliação da mobilidade velar em indivíduos com insuficiência velofaríngea por rinometria acústica, realizada por Bruna Mara Adorno Marmontel Araújo $\mathrm{n}^{\circ}$ do Conselho: CRFA 9293, sob a orientação da Dra. Inge Elly Kiemle Trindade CRBM: 1-2081, que tem como objetivo verificar se a rinometria acústica é capaz de identificar a deficiência no movimento velar que caracteriza a Insuficiência Velofaríngea.

No laboratório de Fisiologia do Hospital de Reabilitação das Anomalias Craniofaciais (CENTRINHO), você (seu filho) passará por uma avaliação fonoaudiológica, usada de rotina em pacientes atendidos nesse setor, e será submetido a um exame chamado "Rinometria Acústica". Durante esse exame, o paciente fica sentado com queixo apoiado em um suporte, um tubo do equipamento é encostado na narina e um som é lançado no nariz por meio desse tubo e recolhido, posteriormente, como um eco. A análise desse eco com o auxílio de um computador permite medir o tamanho do espaço aéreo dentro do nariz e verificar como estão funcionando as estruturas que separam a boca do nariz durante a respiração e durante a fala. $O$ exame não causa nenhuma dor, apenas um pequeno desconforto e será realizado duas vezes, antes e 10 minutos após o uso de um descongestionante nasal.

Na divulgação dos dados da pesquisa, a sua identidade (do seu filho) será mantida em sigilo. Qualquer dúvida poderá ser por nós esclarecida pessoalmente ou pelo telefone (14) 3235-8137.

"Caso o sujeito da pesquisa queira apresentar reclamações em relação a sua participação na pesquisa, poderá entrar em contato com o Comitê de Ética em Pesquisa em Seres Humanos, do HRAC-USP, pelo endereço Rua Silvio Marchione, 3-20 no Serviço de Apoio ao Ensino, Pesquisa e Extensão ou pelo telefone (14) 3235-8421".

Fica claro que o sujeito da pesquisa ou seu representante legal pode a qualquer momento retirar seu CONSENTIMENTO LIVRE E ESCLARECIDO e deixar de participar desta pesquisa e ciente de que todas as informações prestadas tornar-se-ão confidenciais e guardadas por força de sigilo profissional (Art. $13^{\circ}$ do Código de Ética de Fonoaudiologia)

Por estarem de acordo assinam o presente termo

Bauru-SP, de de

ou responsável

* A SER PREENCHIDO, SE O SUJEITO DA PESQUISA NÃo FOR O PACIENTE.

Nome do Pesquisador Responsável: Bruna Mara Adorno Marmontel Araújo

Endereço do Pesquisador Responsável (Rua, $\mathrm{N}^{\circ}$ ): Rua Carlos Marques, 17-35

Cidade: Bauru Estado: SP CEP: 17060-230

Telefone: (14) 3232-5382 E-mail: brunaaraujo@usp.br

Endereço Institucional (Rua, No): Rua Silvio Marchione, 3-20

Cidade: Bauru Estado: SP CEP: 17012-900 